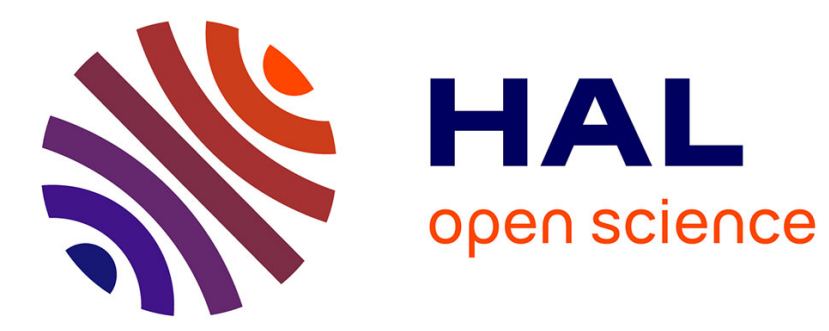

\title{
Sensitivity analysis of 3-RPR planar parallel manipulators
}

Stéphane Caro, Nicolas Binaud, Philippe Wenger

\section{To cite this version:}

Stéphane Caro, Nicolas Binaud, Philippe Wenger. Sensitivity analysis of 3-RPR planar parallel manipulators. Journal of Mechanical Design, 2009, 131, pp.121005-1-121005-13. 10.1115/1.4000216 . hal-00456135v2

\section{HAL Id: hal-00456135 \\ https://hal.science/hal-00456135v2}

Submitted on 14 Mar 2010

HAL is a multi-disciplinary open access archive for the deposit and dissemination of scientific research documents, whether they are published or not. The documents may come from teaching and research institutions in France or abroad, or from public or private research centers.
L'archive ouverte pluridisciplinaire HAL, est destinée au dépôt et à la diffusion de documents scientifiques de niveau recherche, publiés ou non, émanant des établissements d'enseignement et de recherche français ou étrangers, des laboratoires publics ou privés. 


\title{
SENSITIVITY ANALYSIS OF 3-RPR PLANAR PARALLEL MANIPULATORS
}

\author{
Stéphane Caro, Nicolas Binaud, Philippe Wenger \\ Institut de Recherche en Communications et Cybernétique de Nantes \\ UMR CNRS n 6597 \\ 1 rue de la Noë, 44321 Nantes, France \\ Email: \{stephane.caro, nicolas.binaud, philippe.wenger\}@irccyn.ec-nantes.fr
}

\begin{abstract}
This paper deals with the sensitivity analysis of 3-Rㅛ planar parallel manipulators (PPMs). First, the sensitivity coefficients of the pose of the manipulator moving platform to variations in the geometric parameters and in the actuated variables are expressed algebraically. Moreover, two aggregate sensitivity indices are determined, one related to the orientation of the manipulator moving platform and another one related to its position. Then, a methodology is proposed to compare 3-RPR PPMs with regard to their dexterity, workspace size and sensitivity. Finally, the sensitivity of a 3-R $\underline{P R}$ PPM is analyzed in detail and four 3-Rㅛ RPMs are compared as illustrative examples.
\end{abstract}

\section{NOMENCLATURE}

$a_{i} \quad$ Distance between points $O$ and $A_{i}$

$\rho_{i} \quad$ Distance between points $A_{i}$ and $C_{i}$

$c_{i} \quad$ Distance between points $C_{i}$ and $P$

$\alpha_{i} \quad$ Angle between vectors $\overrightarrow{O x}$ and $\overrightarrow{O A}_{i}$

$\beta_{i} \quad$ Angle between vectors $\overrightarrow{C_{1} C_{2}}$ and $\overrightarrow{P C_{i}}$

$\theta_{i} \quad$ Angle between vectors $\overrightarrow{O x}$ and $\overrightarrow{A_{i} C_{i}}$ 
$\delta a_{i} \quad$ Variation in $a_{i}$

$\delta \alpha_{i} \quad$ Variation in $\alpha_{i}$

$\delta \rho_{i} \quad$ Variation in $\rho_{i}$

$\delta c_{i} \quad$ Variation in $c_{i}$

$\delta \beta_{i} \quad$ Variation in $\beta_{i}$

$\|\cdot\|_{2} \quad$ The Euclidean norm

$\mathbf{h}_{i} \quad$ Unit vector $\overrightarrow{O A}_{i} /\left\|\overrightarrow{O A}_{i}\right\|_{2}$

$\mathbf{u}_{i} \quad$ Unit vector $\overrightarrow{A_{i} C_{i}} /\left\|\overrightarrow{A_{i} C_{i}}\right\|_{2}$

$\mathbf{k}_{i} \quad$ Unit vector $\overrightarrow{C_{i} P} /\left\|\overrightarrow{C_{i} P}\right\|_{2}$

$\mathcal{F}_{b} \quad$ Base frame

$\mathcal{F}_{p} \quad$ Moving platform frame

$P \quad$ Geometric center of the moving platform

$p_{x}, p_{y} \quad$ Cartesian coordinates of $P$ expressed in $\mathcal{F}_{b}$

$\phi$ Orientation of the moving platform

$\delta a_{i x} \quad$ Position error of point $A_{i}$ along $\overrightarrow{O x}$

$\delta a_{i y} \quad$ Position error of point $A_{i}$ along $\overrightarrow{O y}$

$\delta c_{i X} \quad$ Position error of point $C_{i}$ along $\overrightarrow{P X}$

$\delta c_{i Y} \quad$ Position error of point $C_{i}$ along $\overrightarrow{P Y}$

$v_{p} \quad$ Local sensitivity index of the position of the moving platform to variations in the geometric parameters

$v_{\phi} \quad$ Local sensitivity index of the orientation of the moving platform to variations in the geometric parameters

\section{INTRODUCTION}

Variations in the geometric parameters of PKMs can be either compensated or amplified. For that reason, it is important to analyze the sensitivity of the mechanism performance to variations in its geometric parameters. For instance, Wang et al. [1] studied the effect of manufacturing tolerances on the accuracy of a Stewart platform. Kim et al. [2] used a forward error bound analysis to find the error bound of the end-effector of a Stewart platform when the error bounds of the joints are given, and an inverse error bound analysis to determine those of the joints for the given error bound of the end-effector. Kim and Tsai [3] studied the effect of misalignment of linear actuators of a 3-Degree of Freedom (DOF) translational 
parallel manipulator on the motion of its moving platform. Caro et al. [4] developed a tolerance synthesis method for mechanisms based on a robust design approach. Caro et al. [巨] proposed two indices to evaluate the sensitivity of the endeffector pose (position + orientation) of Orthoglide 3-axis, a 3-DOF translational PKM, to variations in its design parameters. Besides, they noticed that the better the dexterity, the higher the accuracy of the manipulator. However, Yu et al. [6] claimed that the accuracy of a 3-DOF Planar Parallel Manipulator (PPM) is not necessarily related to its dexterity. Meng et al. [T] proposed a method to analyze the accuracy of parallel manipulators with joint clearance and obtained a standard convex optimization problem to evaluate the maximal pose error in a prescribed workspace.

This paper deals with the sensitivity analysis of 3-DOF Planar Parallel Manipulators (PPMs) to variations in their geometric parameters and actuated joints. Without loss of generality, we focus on the sensitivity analysis of the 3-R $\underline{P} R$ manipulator within the framework of this paper. The singularities of this manipulator were analyzed in [9, 10]. Here, we introduce a methodology to derive the sensitivity coefficients of the moving platform pose to variations in the geometric parameters in algebraic form. The underlying methodology can also be applied to derive the sensitivity coefficients of other PPMs such as

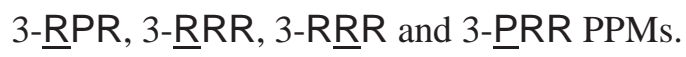

First, the architecture of the manipulator is described. Then, the sensitivity coefficients of the moving platform pose to variations in the geometric parameters and in the prismatic actuated variables are expressed algebraically. Moreover, two aggregate sensitivity indices are determined, one related to the orientation of the manipulator moving platform and another one related to its position. Then, a methodology is proposed to compare 3-RPR PPMs with regard to their dexterity, workspace size and sensitivity. Finally, the sensitivity of an arbitrary 3-R $\underline{P R}$ PPM is analyzed in detail and four 3-R $\underline{P} R$ PPMs are compared as illustrative examples.

\section{MANIPULATOR ARCHITECTURE}

Here and throughout this paper, R, P and $\underline{P}$ denote revolute, prismatic and actuated prismatic joints, respectively. Figure 11 illustrates the architecture of the manipulator under study. It is composed of a base and a moving platform (MP) connected by means of three legs. Points $A_{1}, A_{2}$ and $A_{3},\left(C_{1}, C_{2}\right.$ and $C_{3}$, respectively) lie at the corners of a triangle, of which point $O$ (point $P$, resp.) is the circumcenter. Each leg is composed of a $\mathrm{R}$, a $\mathrm{P}$ and a $\mathrm{R}$ joint in sequence. The three $\mathrm{P}$ joints are actuated. Accordingly, the manipulator is named 3-RPR manipulator.

$\mathcal{F}_{b}$ and $\mathcal{F}_{p}$ are the base and the moving platform frames of the manipulator. In the scope of this paper, $\mathcal{F}_{b}$ and $\mathcal{F}_{p}$ are supposed to be orthogonal. $\mathcal{F}_{b}$ is defined with the orthogonal dihedron $(\overrightarrow{O x}, \overrightarrow{O y})$, point $O$ being its center and $\overrightarrow{O x}$ parallel to 
segment $A_{1} A_{2}$. Likewise, $\mathcal{F}_{p}$ is defined with the orthogonal dihedron $(\overrightarrow{P X}, \overrightarrow{P Y})$, point $C$ being its center and $\overrightarrow{P X}$ parallel to segment $C_{1} C_{2}$.

The manipulator MP pose, i.e., its position and its orientation, is determined by means of the Cartesian coordinates vector $\mathbf{p}=\left[p_{x}, p_{y}\right]^{T}$ of operation point $P$ expressed in frame $\mathcal{F}_{b}$ and angle $\phi$, namely, the angle between frames $\mathcal{F}_{b}$ and $\mathcal{F}_{p}$. Finally, the passive joints do not have any stop.

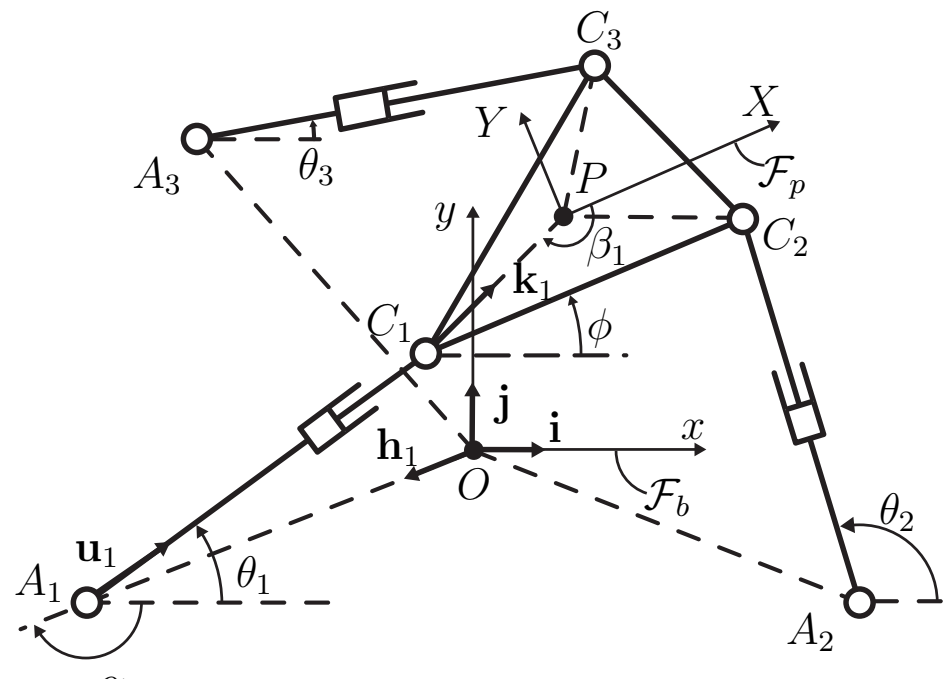

Figure 1. 3-RPR manipulator

\section{SENSITIVITY INDICES}

In this section, we first derive the sensitivity coefficients of the pose of the 3-R $\underline{P R}$ manipulator MP to variations in the prismatic actuated joints as well as in the coordinates of $A_{i}$ and $C_{i}, i=1,2,3$, the latter being either Polar or Cartesian coordinates. From the foregoing sensitivity coefficients, we propose sensitivity indices associated with the variations in the coordinates of $A_{i}, C_{i}$ and in $\rho_{i}, i=1,2,3$, and two aggregate sensitivity indices, one related to the position of the MP of the manipulator and another one related to its orientation. 


\subsection{Sensitivity Coefficients}

From the closed-loop kinematic chains $O-A_{i}-C_{i}-P-O, i=1, \ldots, 3$ depicted in Fig. 1, the position vector $\mathbf{p}$ of point $P$ can be expressed in $\mathcal{F}_{b}$ as follows:

$$
\mathbf{p}=\left[\begin{array}{l}
p_{x} \\
p_{y}
\end{array}\right]=\mathbf{a}_{i}+\left(\mathbf{c}_{i}-\mathbf{a}_{i}\right)+\left(\mathbf{p}-\mathbf{c}_{i}\right), \quad i=1, \ldots, 3
$$

$\mathbf{a}_{i}$ and $\mathbf{c}_{i}$ being the position vectors of points $A_{i}$ and $C_{i}$ expressed in $\mathcal{F}_{b}$. Equation (1) can also be written as

$$
\mathbf{p}=a_{i} \mathbf{h}_{i}+\rho_{i} \mathbf{u}_{i}+c_{i} \mathbf{k}_{i}
$$

with

$$
\mathbf{h}_{i}=\left[\begin{array}{c}
\cos \alpha_{i} \\
\sin \alpha_{i}
\end{array}\right], \mathbf{u}_{i}=\left[\begin{array}{c}
\cos \theta_{i} \\
\sin \theta_{i}
\end{array}\right], \mathbf{k}_{i}=\left[\begin{array}{c}
\cos \left(\phi+\beta_{i}+\pi\right) \\
\sin \left(\phi+\beta_{i}+\pi\right)
\end{array}\right]
$$

where $a_{i}$ is the distance between points $O$ and $A_{i}, \rho_{i}$ is the distance between points $A_{i}$ and $C_{i}, c_{i}$ is the distance between points $C_{i}$ and $P, \mathbf{h}_{i}$ is the unit vector $\overrightarrow{O A_{i}} /\left\|\overrightarrow{O A_{i}}\right\|_{2}$, $\mathbf{u}_{i}$ is the unit vector $\overrightarrow{A_{i} C_{i}} /\left\|\overrightarrow{A_{i} C_{i}}\right\|_{2}$ and $\mathbf{k}_{i}$ is the unit vector $\overrightarrow{C_{i} P} /\left\|\overrightarrow{C_{i} P}\right\|_{2}$.

Upon differentiation of Eq. (2), we obtain:

$$
\begin{aligned}
\delta \mathbf{p}= & \delta a_{i} \mathbf{h}_{i}+a_{i} \delta \alpha_{i} \mathbf{E} \mathbf{h}_{i}+\delta \rho_{i} \mathbf{u}_{i}+\rho_{i} \delta \theta_{i} \mathbf{E} \mathbf{u}_{i} \\
& +\delta c_{i} \mathbf{k}_{i}+c_{i}\left(\delta \phi+\delta \beta_{i}\right) \mathbf{E} \mathbf{k}_{i}
\end{aligned}
$$


with matrix $\mathbf{E}$ defined as

$$
\mathbf{E}=\left[\begin{array}{cc}
0 & -1 \\
1 & 0
\end{array}\right]
$$

$\delta \mathbf{p}$ and $\delta \phi$ being the position and orientation errors of the MP. Likewise, $\delta a_{i}, \delta \alpha_{i}, \delta \rho_{i}, \delta c_{i}$ and $\delta \beta_{i}$ denote the variations in $a_{i}$, $\alpha_{i}, \rho_{i}, c_{i}$ and $\beta_{i}$, respectively.

The idle variation $\delta \theta_{i}$ is eliminated by dot-multiplying Eq.(3) by $\rho_{i} \mathbf{u}_{i}^{T}$, thus obtaining

$$
\begin{aligned}
\rho_{i} \mathbf{u}_{i}^{T} \delta \mathbf{p}= & \rho_{i} \delta a_{i} \mathbf{u}_{i}^{T} \mathbf{h}_{i}+\rho_{i} a_{i} \delta \alpha_{i} \mathbf{u}_{i}^{T} \mathbf{E} \mathbf{h}_{i}+\rho_{i} \delta \rho_{i} \\
& +\rho_{i} \delta c_{i} \mathbf{u}_{i}^{T} \mathbf{k}_{i}+\rho_{i} c_{i}\left(\delta \phi+\delta \beta_{i}\right) \mathbf{u}_{i}^{T} \mathbf{E} \mathbf{k}_{i}
\end{aligned}
$$

Equation (5) can now be cast in vector form, namely,

$$
\begin{gathered}
\mathbf{A}\left[\begin{array}{l}
\delta \phi \\
\delta \mathbf{p}
\end{array}\right]=\mathbf{H}_{a}\left[\begin{array}{l}
\delta a_{1} \\
\delta a_{2} \\
\delta a_{3}
\end{array}\right]+\mathbf{H}_{\alpha}\left[\begin{array}{l}
\delta \alpha_{1} \\
\delta \alpha_{2} \\
\delta \alpha_{3}
\end{array}\right]+\mathbf{B}\left[\begin{array}{l}
\delta \rho_{1} \\
\delta \rho_{2} \\
\delta \rho_{3}
\end{array}\right]+ \\
\mathbf{H}_{c}\left[\begin{array}{l}
\delta c_{1} \\
\delta c_{2} \\
\delta c_{3}
\end{array}\right]+\mathbf{H}_{\beta}\left[\begin{array}{l}
\delta \beta_{1} \\
\delta \beta_{2} \\
\delta \beta_{3}
\end{array}\right]
\end{gathered}
$$

with

$$
\mathbf{A}=\left[\begin{array}{ll}
m_{1} & \rho_{1} \mathbf{u}_{1}^{T} \\
m_{2} & \rho_{2} \mathbf{u}_{2}^{T} \\
m_{3} & \rho_{3} \mathbf{u}_{3}^{T}
\end{array}\right], \mathbf{B}=\left[\begin{array}{ccc}
\rho_{1} & 0 & 0 \\
0 & \rho_{2} & 0 \\
0 & 0 & \rho_{3}
\end{array}\right]
$$




$$
\begin{aligned}
& \mathbf{H}_{a}=\operatorname{diag}\left[\rho_{1} \mathbf{u}_{1}^{T} \mathbf{h}_{1} \rho_{2} \mathbf{u}_{2}^{T} \mathbf{h}_{2} \rho_{3} \mathbf{u}_{3}^{T} \mathbf{h}_{3}\right] \\
& \mathbf{H}_{\alpha}=\operatorname{diag}\left[\rho_{1} a_{1} \mathbf{u}_{1}^{T} \mathbf{E} \mathbf{h}_{1} \rho_{2} a_{2} \mathbf{u}_{2}^{T} \mathbf{E} \mathbf{h}_{2} \rho_{3} a_{3} \mathbf{u}_{3}^{T} \mathbf{E} \mathbf{h}_{3}\right] \\
& \mathbf{H}_{c}=\operatorname{diag}\left[\rho_{1} \mathbf{u}_{1}^{T} \mathbf{k}_{1} \rho_{2} \mathbf{u}_{2}^{T} \mathbf{k}_{2} \rho_{3} \mathbf{u}_{3}^{T} \mathbf{k}_{3}\right] \\
& \mathbf{H}_{\beta}=\operatorname{diag}\left[\rho_{1} c_{1} \mathbf{u}_{1}^{T} \mathbf{E} \mathbf{k}_{1} \rho_{2} c_{2} \mathbf{u}_{2}^{T} \mathbf{E} \mathbf{k}_{2} \rho_{3} c_{3} \mathbf{u}_{3}^{T} \mathbf{E} \mathbf{k}_{3}\right]
\end{aligned}
$$

and

$$
m_{i}=-\rho_{i} c_{i} \mathbf{u}_{i}^{T} \mathbf{E k}_{i}, \quad i=1, \ldots, 3
$$

Let us notice that $\mathbf{A}$ and $\mathbf{B}$ are the direct and the inverse Jacobian matrices of the manipulator, respectively. Assuming that $\mathbf{A}$ is non singular, i.e., the manipulator does not meet any Type II singularity [11], we obtain upon multiplication of Eq.(可) by $\mathbf{A}^{-1}$ :

$$
\begin{aligned}
& {\left[\begin{array}{c}
\delta \phi \\
\delta \mathbf{p}
\end{array}\right]=} \mathbf{J}_{a}\left[\begin{array}{l}
\delta a_{1} \\
\delta a_{2} \\
\delta a_{3}
\end{array}\right]+\mathbf{J}_{\alpha}\left[\begin{array}{l}
\delta \alpha_{1} \\
\delta \alpha_{2} \\
\delta \alpha_{3}
\end{array}\right]+\mathbf{J}\left[\begin{array}{l}
\delta \rho_{1} \\
\delta \rho_{2} \\
\delta \rho_{3}
\end{array}\right]+ \\
& \mathbf{J}_{c}\left[\begin{array}{l}
\delta c_{1} \\
\delta c_{2} \\
\delta c_{3}
\end{array}\right]+\mathbf{J}_{\beta}\left[\begin{array}{l}
\delta \beta_{1} \\
\delta \beta_{2} \\
\delta \beta_{3}
\end{array}\right]
\end{aligned}
$$

with

$$
\begin{gathered}
\mathbf{J}=\mathbf{A}^{-1} \mathbf{B} \\
\mathbf{J}_{a}=\mathbf{A}^{-1} \mathbf{H}_{a} \\
\mathbf{J}_{\alpha}=\mathbf{A}^{-1} \mathbf{H}_{\alpha}
\end{gathered}
$$




$$
\begin{aligned}
\mathbf{J}_{c} & =\mathbf{A}^{-1} \mathbf{H}_{c} \\
\mathbf{J}_{\beta} & =\mathbf{A}^{-1} \mathbf{H}_{\beta}
\end{aligned}
$$

and

$$
\begin{aligned}
\mathbf{A}^{-1} & =\frac{1}{\operatorname{det}(\mathbf{A})}\left[\begin{array}{lll}
v_{1} & v_{2} & v_{3} \\
\mathbf{v}_{1} & \mathbf{v}_{2} & \mathbf{v}_{3}
\end{array}\right] \\
v_{i} & =\rho_{j} \rho_{k}\left(\mathbf{u}_{j} \times \mathbf{u}_{k}\right)^{T} \mathbf{k} \\
\mathbf{v}_{i} & =\mathbf{E}\left(m_{j} \rho_{k} \mathbf{u}_{k}-m_{k} \rho_{j} \mathbf{u}_{j}\right) \\
\operatorname{det}(\mathbf{A}) & =\sum_{i=1}^{3} m_{i} v_{i} \\
\mathbf{k} & =\mathbf{i} \times \mathbf{j}
\end{aligned}
$$

$j=(i+1)$ modulo 3; $k=(i+2)$ modulo 3; $i=1,2,3 . \mathbf{J}$ is the kinematic Jacobian matrix of the manipulator whereas $\mathbf{J}_{a}, \mathbf{J}_{\alpha}$, $\mathbf{J}_{c}$ and $\mathbf{J}_{\beta}$ are named sensitivity Jacobian matrices of the pose of the MP to variations in $a_{i}, \alpha_{i}, c_{i}$ and $\beta_{i}$, respectively. Indeed, the terms of $\mathbf{J}_{a}, \mathbf{J}_{\alpha}, \mathbf{J}_{c}$ and $\mathbf{J}_{\beta}$ are the sensitivity coefficients of the position and the orientation of the moving platform of the manipulator to variations in the Polar coordinates of points $A_{i}$ and $C_{i}$. Likewise, $\mathbf{J}$ contains the sensitivity coefficients of the manipulator MP pose to variations in the prismatic actuated joints. It is noteworthy that all these sensitivity coefficients are expressed algebraically.

Let $\delta a_{i x}$ and $\delta a_{i y}$ denote the position errors of points $A_{i}, i=1,2,3$, along $\overrightarrow{O x}$ and $\overrightarrow{O y}$, namely, the variations in the Cartesian coordinates of points $A_{i}$. Likewise, let $\delta c_{i X}$ and $\delta c_{i Y}$ denote the position errors of points $C_{i}$ along $\overrightarrow{P X}$ and $\overrightarrow{P Y}$, namely, the variations in the Cartesian coordinates of points $C_{i}$.

From Fig. 1,

$$
\left[\begin{array}{l}
\delta a_{i x} \\
\delta a_{i y}
\end{array}\right]=\left[\begin{array}{cc}
\cos \alpha_{i} & -a_{i} \sin \alpha_{i} \\
\sin \alpha_{i} & a_{i} \cos \alpha_{i}
\end{array}\right]\left[\begin{array}{l}
\delta a_{i} \\
\delta \alpha_{i}
\end{array}\right]
$$




$$
\left[\begin{array}{l}
\delta c_{i X} \\
\delta c_{i Y}
\end{array}\right]=\left[\begin{array}{cc}
\cos \beta_{i} & -c_{i} \sin \beta_{i} \\
\sin \beta_{i} & c_{i} \cos \beta_{i}
\end{array}\right]\left[\begin{array}{l}
\delta c_{i} \\
\delta \beta_{i}
\end{array}\right]
$$

Accordingly, from Eq.(9) and Eqs.(12a)-(b), we obtain the following relation between the pose error of the MP and variations in the Cartesian coordinates of points $A_{i}$ and $C_{i}$ :

$$
\left[\begin{array}{l}
\delta \phi \\
\delta \mathbf{p}
\end{array}\right]=\mathbf{J}_{A}\left[\begin{array}{l}
\delta a_{1 x} \\
\delta a_{1 y} \\
\delta a_{2 x} \\
\delta a_{2 y} \\
\delta a_{3 x} \\
\delta a_{3 y}
\end{array}\right]+\mathbf{J}\left[\begin{array}{l}
\delta \rho_{1} \\
\delta \rho_{2} \\
\delta \rho_{3}
\end{array}\right]+\mathbf{J}_{C}\left[\begin{array}{l}
\delta c_{1 X} \\
\delta c_{1 Y} \\
\delta c_{2 X} \\
\delta c_{2 Y} \\
\delta c_{3 X} \\
\delta c_{3 Y}
\end{array}\right]
$$

$\mathbf{J}_{A}$ and $\mathbf{J}_{C}$ being named sensitivity Jacobian matrices of the pose of the MP to variations in the Cartesian coordinates of points $A_{i}$ and $C_{i}$, respectively. Indeed, the terms of $\mathbf{J}_{A}$ and $\mathbf{J}_{C}$ are the sensitivity coefficients of the pose of the MP to variations in the Cartesian coordinates of points $A_{i}$ and $C_{i}$.

In order to better highlight the sensitivity coefficients, let us write the $3 \times 6$ matrices $\mathbf{J}_{A}$ and $\mathbf{J}_{C}$ and the $3 \times 3$ matrix $\mathbf{J}$ as follows:

$$
\begin{aligned}
& \mathbf{J}_{A}=\left[\begin{array}{lll}
\mathbf{J}_{A_{1}} & \mathbf{J}_{A_{2}} & \mathbf{J}_{A_{3}}
\end{array}\right] \\
& \mathbf{J}_{C}=\left[\begin{array}{lll}
\mathbf{J}_{C_{1}} & \mathbf{J}_{C_{2}} & \mathbf{J}_{C_{3}}
\end{array}\right] \\
& \mathbf{J}=\left[\mathbf{j}_{1} \mathbf{j}_{2} \mathbf{j}_{3}\right]
\end{aligned}
$$


the $3 \times 2$ matrices $\mathbf{J}_{A_{i}}$ and $\mathbf{J}_{C_{i}}$ and the three dimensional vectors $\mathbf{j}_{i}$ being expressed as:

$$
\begin{aligned}
\mathbf{J}_{A_{i}} & =\left[\begin{array}{l}
\mathbf{j}_{A_{i} \phi} \\
\mathbf{J}_{A_{i} p}
\end{array}\right], \quad i=1,2,3 \\
\mathbf{J}_{C_{i}} & =\left[\begin{array}{l}
\mathbf{j}_{C_{i} \phi} \\
\mathbf{J}_{C_{i} p}
\end{array}\right], \quad i=1,2,3 \\
\mathbf{j}_{i} & =\left[\begin{array}{l}
j_{i \phi} \\
\mathbf{j}_{i p}
\end{array}\right], \quad i=1,2,3
\end{aligned}
$$

with

$$
\begin{aligned}
& \mathbf{j}_{A_{i} \phi}=\frac{1}{\operatorname{det}(\mathbf{A})}\left[v_{i} q_{i} v_{i} r_{i}\right] \\
& \mathbf{j}_{C_{i} \phi}=\frac{1}{\operatorname{det}(\mathbf{A})}\left[v_{i} s_{i} v_{i} t_{i}\right] \\
& j_{i \phi}=\frac{\rho_{i} v_{i}}{\operatorname{det}(\mathbf{A})} \\
& \mathbf{J}_{A_{i} p}=\frac{1}{\operatorname{det}(\mathbf{A})}\left[\begin{array}{lll}
q_{i} \mathbf{v}_{i}^{T} & \mathbf{i} & r_{i} \mathbf{v}_{i}^{T} \mathbf{i} \\
q_{i} \mathbf{v}_{i}^{T} & \mathbf{j} & r_{i} \mathbf{v}_{i}^{T} \mathbf{j}
\end{array}\right] \\
& \mathbf{J}_{C_{i} p}=\frac{1}{\operatorname{det}(\mathbf{A})}\left[\begin{array}{c}
s_{i} \mathbf{v}_{i}^{T} \mathbf{i} t_{i} \mathbf{v}_{i}^{T} \mathbf{i} \\
s_{i} \mathbf{v}_{i}^{T} \mathbf{j} t_{i} \mathbf{v}_{i}^{T} \mathbf{j}
\end{array}\right] \\
& \mathbf{j}_{i p}=\frac{1}{\operatorname{det}(\mathbf{A})}\left[\begin{array}{c}
\rho_{i} \mathbf{v}_{i}^{T} \mathbf{i} \\
\rho_{i} \mathbf{v}_{i}^{T} \mathbf{j}
\end{array}\right]
\end{aligned}
$$

$q_{i}, r_{i}, s_{i}$ and $t_{i}$ taking the form:

$$
\begin{aligned}
q_{i} & =\rho_{i} \mathbf{u}_{i}^{T} \mathbf{i} \\
r_{i} & =\rho_{i} \mathbf{u}_{i}^{T} \mathbf{j}
\end{aligned}
$$




$$
\begin{aligned}
s_{i} & =\rho_{i} \mathbf{u}_{i}^{T} \mathbf{k}_{i} \cos \beta_{i}-\rho_{i} \mathbf{u}_{i}^{T} \mathbf{E} \mathbf{k}_{i} \sin \beta_{i} \\
t_{i} & =\rho_{i} \mathbf{u}_{i}^{T} \mathbf{k}_{i} \sin \beta_{i}+\rho_{i} \mathbf{u}_{i}^{T} \mathbf{E} \mathbf{k}_{i} \cos \beta_{i}
\end{aligned}
$$

$\mathbf{j}_{A_{i} \phi}, \mathbf{j}_{C_{i} \phi}$ and $j_{i \phi}$ contain the sensitivity coefficients of the orientation of the MP of the manipulator to variations in the Cartesian coordinates of points $A_{i}, C_{i}$ and in prismatic actuated variables, respectively. Similarly, $\mathbf{J}_{A_{i} p}, \mathbf{J}_{C_{i} p}$ and $\mathbf{j}_{i p}$ contain the sensitivity coefficients related to the position of the MP.

Accordingly, the designer of such a planar parallel manipulator can easily identify the most influential geometric variations to the pose of its MP and synthesize proper dimensional tolerances from the previous sensitivity coefficients. Some sensitivity indices related to the geometric errors of the moving and base platforms as well as to prismatic actuated joints errors are introduced thereafter.

\subsection{Sensitivity Indices to Variations in the Cartesian Coordinates of $A_{i}, C_{i}$ and in $\rho_{i}$}

From Eqs.16a)-(c) (Eqs.16d)-(f), resp.), it turns out that the maximum sensitivity of the orientation (position, resp.) of the manipulator MP to variations in the Cartesian coordinates of points $A_{i}, C_{i}$ and in $\rho_{i}$ is equal to the norm of $\mathbf{j}_{A_{i} \phi}, \mathbf{j}_{C_{i} \phi}$ and $j_{i \phi}\left(\mathbf{J}_{A_{i} p}, \mathbf{J}_{A_{i} p}\right.$ and $\mathbf{j}_{i p}$, resp.). Accordingly, let $v_{\phi A_{i}}, v_{\phi C_{i}}$ and $v_{\phi \rho_{i}}\left(v_{p A_{i}}, v_{p \rho_{i}}\right.$ and $v_{p C_{i}}$, resp. $)$ be the sensitivity indices of the orientation (position, resp.) of the moving platform to variations in the Cartesian coordinates of points $A_{i}, C_{i}$ and in $\rho_{i}$, respectively:

$$
\begin{aligned}
v_{\phi A_{i}} & =\left\|\mathbf{j}_{A_{i} \phi}\right\|_{2} \\
v_{\phi C_{i}} & =\left\|\mathbf{j}_{C_{i} \phi}\right\|_{2} \\
v_{\phi \rho_{i}} & =\left|j_{i \phi}\right| \\
v_{p A_{i}} & =\left\|\mathbf{J}_{A_{i} p}\right\|_{2} \\
v_{p C_{i}} & =\left\|\mathbf{J}_{C_{i} p}\right\|_{2} \\
v_{p \rho_{i}} & =\left\|\mathbf{j}_{i p}\right\|_{2}
\end{aligned}
$$

with $\|\cdot\|_{2}$ denoting the spectral norm, i.e., the 2-norm. As a reminder, the spectral norm of a matrix is equal to its maximum singular value. 


\subsection{Two Aggregate Sensitivity Indices}

The pose errors of the manipulator MP depend on variations in the geometric parameters as well as on the manipulator configuration. In order to analyze the influence of the manipulator configuration on those errors, let us first formulate some indices to assess the aggregate sensitivity of the MP pose to variations in the geometric parameters for a given manipulator configuration. To this end, let Eq. $(13)$ be expressed as:

$$
\left[\begin{array}{c}
\delta \phi \\
\delta \mathbf{p}
\end{array}\right]=\mathbf{J}_{s}\left[\delta \mathbf{a}_{i} \delta \rho_{\mathbf{i}} \delta \mathbf{c}_{i}\right]^{T}
$$

with

$$
\mathbf{J}_{s}=\left[\mathbf{J}_{A} \mathbf{J} \mathbf{J}_{C}\right]
$$

and

$$
\begin{aligned}
\delta \mathbf{a}_{i} & =\left[\begin{array}{lll}
\delta a_{1 x} \delta a_{1 y} \delta a_{2 x} \delta a_{2 y} \delta a_{3 x} \delta a_{3 y}
\end{array}\right] \\
\delta \rho_{\mathbf{i}} & =\left[\begin{array}{ll}
\delta \rho_{1} \delta \rho_{2} \delta \rho_{3}
\end{array}\right] \\
\delta \mathbf{c}_{i} & =\left[\begin{array}{lll}
\delta c_{1 X} \delta c_{1 Y} \delta c_{2 X} \delta c_{2 Y} \delta c_{3 X} \delta c_{3 Y}
\end{array}\right]
\end{aligned}
$$

The $3 \times 15$ matrix $\mathbf{J}_{s}$ can be written as follows:

$$
\mathbf{J}_{s}=\left[\begin{array}{l}
\mathbf{j}_{s_{\phi}} \\
\mathbf{J}_{s_{p}}
\end{array}\right]
$$


with

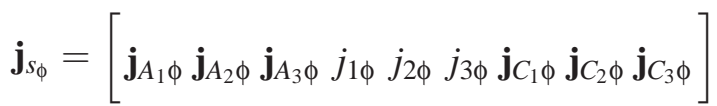

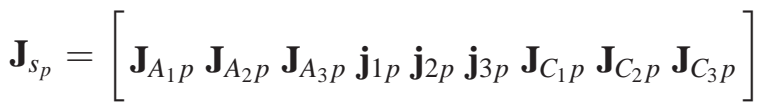

From Eq.23a), we can define an aggregate sensitivity index $v_{\phi}$ of the orientation of the MP of the manipulator to variations in its geometric parameters and prismatic actuated joints, namely,

$$
v_{\phi}=\frac{\left\|\mathbf{j}_{s_{\phi}}\right\|_{2}}{n_{v}}
$$

$n_{v}$ being the number of variations that are considered. Here, $n_{v}$ is equal to 15 .

Likewise, from Eq.(23b), an aggregate sensitivity index $v_{p}$ of the position of the MP of the manipulator to variations in its geometric parameters and prismatic actuated joints can be defined as follows:

$$
\mathrm{v}_{p}=\frac{\left\|\mathbf{J}_{s_{p}}\right\|_{2}}{n_{v}}
$$

For any given manipulator configuration, the lower $v_{\phi}$, the lower the overall sensitivity of the orientation its MP to variations in the geometric parameters. Similarly, the lower $v_{p}$, the lower the overall sensitivity of the MP position to variations in the geometric parameters. As a matter of fact, $v_{\phi}\left(v_{p}\right.$, resp. $)$ characterizes the intrinsic sensitivity of the MP orientation (position, resp.) to any variation in the geometric parameters.

Let us notice that $v_{p}$ as well as the sensitivity coefficients related to the MP position defined in Sections 3.1 and 3.2 are frame dependent, whereas $v_{\phi}$ and the sensitivity coefficients related to the MP orientation are not.

Finally, let us notice that $v_{\phi q_{i}}$ indices, $q_{i}=\left\{A_{i}, \rho_{i}, C_{i}\right\}$, defined in Eqs. 18a)-(c), as well as $v_{\phi}$ are expressed in [rad/L], whereas $v_{p q_{i}}$ indices defined in Eqs.(18d)-(f), as well as $v_{p}$ are dimensionless, [L] being the unit of length. 


\section{COMPARISON METHODOLOGY}

In this section we define a methodology to compare planar parallel manipulators with regard to their dexterity, workspace size and sensitivity. This methodology is organized into four steps:

1. normalization of the geometric parameters;

2. determination of the manipulator regular dexterous workspace (RDW);

3. evaluation of the sensitivity of the MP orientation to variations in the geometric parameters throughout the RDW by means of $v_{\phi}$ defined in Eq. (24);

4. evaluation of the sensitivity of the MP position to variations in the geometric parameters throughout the RDW by means of $v_{p}$ defined in Eq. (25).

The radii of the circumscribed circles of the base and moving platforms of the manipulators are normalized as explained in Section 4.1. The manipulator RDW is obtained by means of an optimization problem introduced in Section 4.2 .

\subsection{Geometric Parameters Normalization}

Let $R_{1}$ and $R_{2}$ be the radii of the base and moving platforms of the PPM. In order to come up with finite values, $R_{1}$ and $R_{2}$ are normalized. In the same vein, the dimensions of two degree-of-freedom manipulators were normalized in [12, 13, 14]. For that matter, let $N_{f}$ be a normalizing factor:

$$
N_{f}=\left(R_{1}+R_{2}\right) / 2
$$

and

$$
r_{m}=R_{m} / N_{f}, \quad m=1,2
$$

Therefore,

$$
r_{1}+r_{2}=2
$$


From eqs. 27) and (28), we can notice that:

$$
r_{1} \in[0,2], r_{2} \in[0,2]
$$

As the former two-dimensional infinite space corresponding to geometric parameters $R_{1}$ and $R_{2}$ is reduced to a one-dimensional finite space defined with Eq.(28), the workspace analysis of the 3-R $\underline{P R}$ manipulator under study is easier. Moreover, once the geometric parameters of two PPMs are normalized, the size of their RDW can be compared.

\subsection{Regular Dexterous Workspace}

Assessing the kinetostatic peformance of parallel manipulators is not an easy task for 6-DOF parallel manipulators [15], but for planar manipulators it is easier as their singularities have a simple geometric interpretation [16, 17].

The regular dexterous workspace of a manipulator (RDW) is a regular-shaped part of its workspace with good and homogeneous kinetostatic performance [18]. The shape of the RDW is up to the designer. It may be a cube, a parallelepiped, a cylinder or another regular shape. A reasonable choice is a shape that "fits well" the one of the singular surfaces. A cylinder suits well for planar manipulators.

In the scope of this study, the RDW of the PPM is supposed to be a cylinder of $\phi$-axis with a good kinetostatic performance, i.e., the inverse condition number $\kappa_{F}^{-1}\left(\mathbf{J}_{n}\right)$ of the normalized Jacobian matrix $\mathbf{J}_{n}$ of the manipulator based on the Frobenius norm is higher than a prescribed value, $\kappa_{F}(\cdot)$ denoting the condition number of a matrix based on the Frobenius norm. Let $\kappa_{F}^{-1}\left(\mathbf{J}_{n}\right)$ be higher than 0.1 .

The normalized Jacobian matrix $\mathbf{J}_{n}$ is used as the terms of the kinematic Jacobian matrix $\mathbf{J}$ are not homogeneous. In this case, its condition number is meaningless as its singular values cannot be arranged in order due to their different nature. $\mathbf{J}_{n}$ is obtained from $\mathbf{J}$ by means of a characteristic length in [16]. For the particular case of planar 3-dof parallel manipulators, the use of the characteristic length to calculate the condition number makes sense as it has a geometric meaning as shown in [17]. Indeed, the characteristic length was calculated such that, at the isotropic configuration, the manipulators is the furthest from its singular configurations, which are those where lines $A_{i} C_{i}$ intersect, $i=1, \ldots, 3$. Here "furthest" does not relate to a distance (there is no metric in $\mathbb{R}^{2} \times S O(2)$ ), but to angles between lines as explained in [17]. A geometric interpretation of the characteristic length was reported in [19]. 
The RDW is obtained by solving the following optimization problem:

$$
P b \mid \begin{aligned}
& \min _{\mathbf{x}} 1 / R \\
& \text { s.t. } \\
& \quad \Delta \phi \geq \pi / 6 \\
& \quad \kappa_{F}^{-1}\left(\mathbf{J}_{n}\right) \geq 0.1
\end{aligned}
$$

$R$ being the radius of the cylinder and $\Delta \phi$ the orientation range of the MP within the RDW. Here, $\Delta \phi$ is supposed to be equal to $\pi / 6$. This optimization problem has five decision variables, namely,

$$
\mathbf{x}=\left[R I_{x} I_{y} \phi_{\min } \phi_{\max }\right]
$$

$I_{x}$ and $I_{y}$ being the Cartesian coordinates of the cylinder center, $\phi_{\min }$ and $\phi_{\max }$ being the lower and upper bounds of $\Delta \phi$, respectively. Besides, the global minimum of this optimization problem is found by means of a Tabu search Hooke and Jeeves algorithm [20]. Consequently, the RDW of the manipulator is completely defined by means of the decision variables associated with this global minimum. Finally, Eqs. (24) and (25) are used to evaluate the overall sensitivity of the MP orientation and position to variations in the geometric parameters of the manipulator throughout the RDW.

\section{ILLUSTRATIVE EXAMPLES}

This section aims at illustrating the sensitivity coefficients, indices and comparison methodology introduced in Sections 3.1, 3.2, 3.3 and 4 , respectively. For that purpose, the sensitivity of an arbitrary 3-RPR PPM is first analyzed in detail. Then, the sensitivity of four 3-RPR PPMs are compared.

\subsection{Sensitivity Analysis of a general 3-RPR PPM}

Let us study the 3-R $\underline{P} R$ PPM with the following geometric parameters:

$$
\begin{aligned}
& a_{1}=a_{2}=a_{3}=R_{1}=0.60 \\
& c_{1}=c_{2}=c_{3}=R_{2}=0.25
\end{aligned}
$$




$$
\begin{array}{r}
\left\{\alpha_{1}, \alpha_{2}, \alpha_{3}\right\}=\{-2.50,-0.60,2.30\} \\
\left\{\beta_{1}, \beta_{2}, \beta_{3}\right\}=\{-2.90,-0.25,0.75\}
\end{array}
$$

$\alpha_{i}$ and $\beta_{i}, i=1,2,3$, being expressed in [rad] and $R_{1}$ and $R_{2}$ in [m]. Figure 6 illustrates the corresponding manipulator, the radii of the circumscribed circles of its base and moving platforms being different. In this figure, the MP orientation $\phi$ is equal to $-\pi / 8$ and the Cartesian coordinates of its geometric center $P$ expressed in $\mathcal{F}_{b}$ are $\mathbf{p}=[-0.3,-0.1]^{T}$. Figure 3

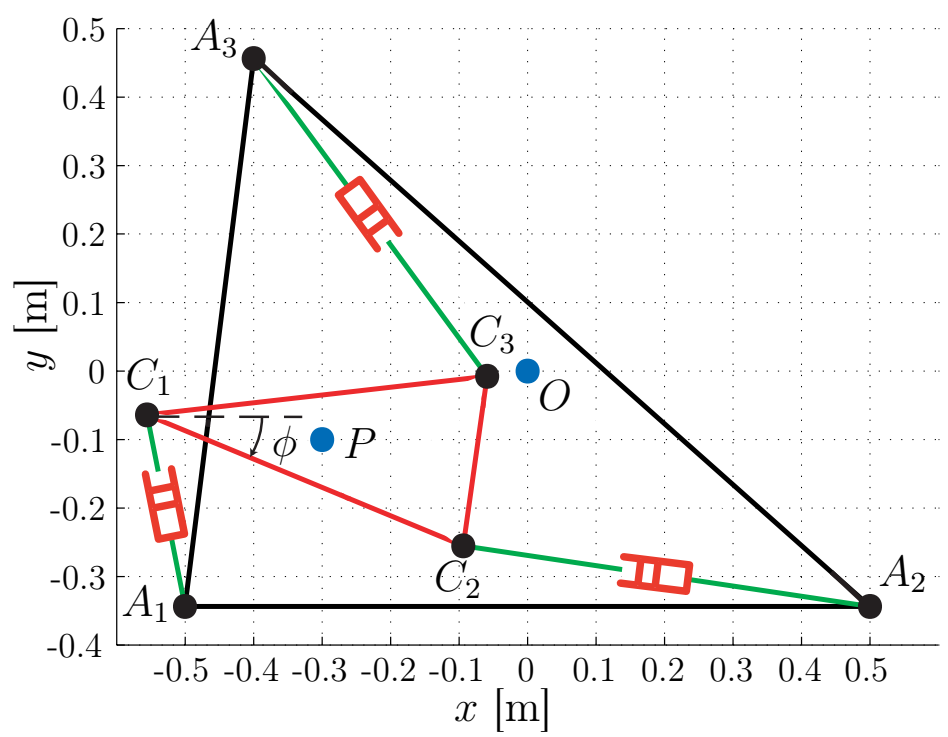

Figure 2. The 3-R $\underline{P R}$ manipulator under study with $\phi=-\pi / 8, \mathbf{p}=[-0.3,-0.1]^{T}, R_{1}=0.6 \mathrm{~m}$ and $R_{2}=0.25 \mathrm{~m}$

illustrates the singularity locus of the 3-Rㅛ PPM within a region of the workspace delimited with $x \in[-2,2], y \in[-2,2]$ and $\phi \in[-2,2]$. They correspond to configurations in which the stiffness of the mechanism is locally lost, which occurs when matrix $\mathbf{A}$ is singular, i.e., $\operatorname{det}(\mathbf{A})=0$, [21].

Let us assume that the prismatic joints of the manipulator do not have any stop. Figure $\theta$ depicts a section of the workspace of the 3-RPR PPM under study for a given orientation of its moving platform, i.e., $\phi=-\pi / 8$. This section, called $\mathcal{W}_{s}$, is an ellipse and is delimited with the singularity locus shown in Fig. 3 .

Figures 5(a) (b) illustrate the percentage of $\mathcal{W}_{s}$, in which the orientation-sensitivity indices related to variations in the coordinates of $A_{i}, C_{i}$ and in $\rho_{i}$, defined with Eqs. (18a)-(c), are smaller than $3 \mathrm{rad} / \mathrm{m}$ and $6 \mathrm{rad} / \mathrm{m}$, respectively. The three bars above indices $v_{\phi q_{i}}, i=1,2,3$, are associated with the sensitivity of the MP orientation $\phi$ to variations in $q_{1}, q_{2}$ and $q_{3}$, 


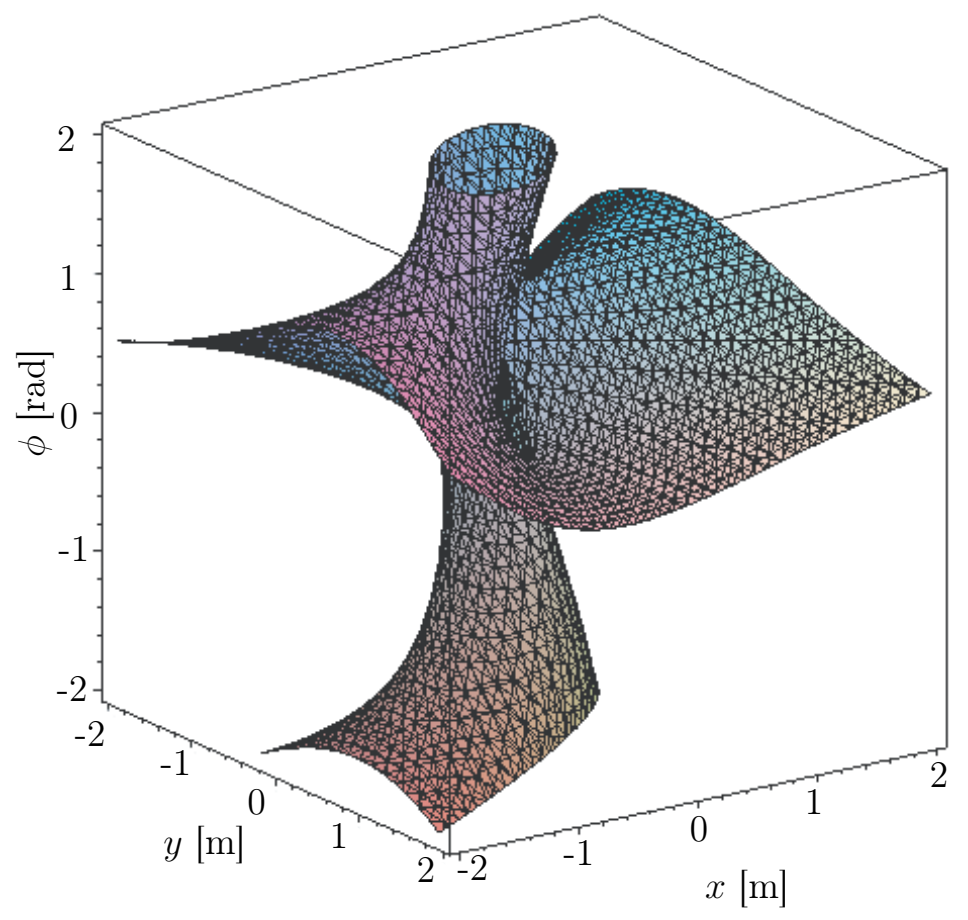

Figure 3. Singularity locus of the 3-RPR PPM within a region of the workspace delimited with $x \in[-2,2], y \in[-2,2]$ and $\phi \in[-2,2]$

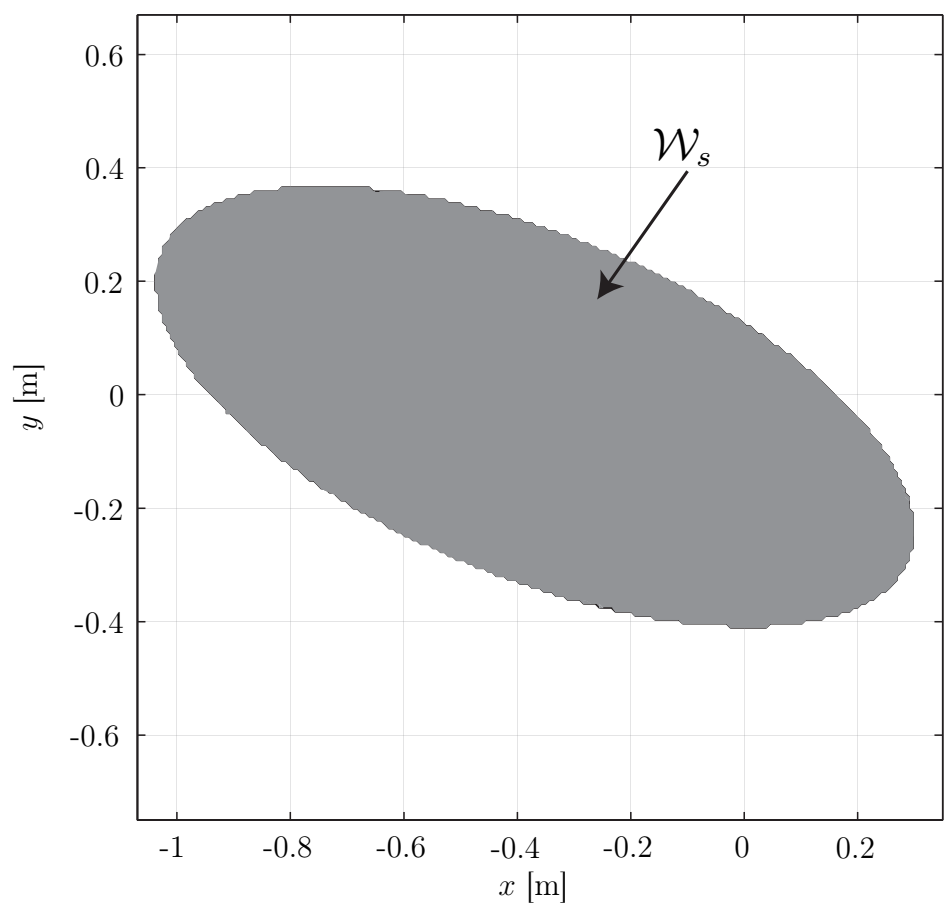

Figure 4. Section of the workspace of the 3-RPR PPM delimited with the Type-II singularities for a given orientation $\phi$, i.e., $\phi=-\pi / 8$ 


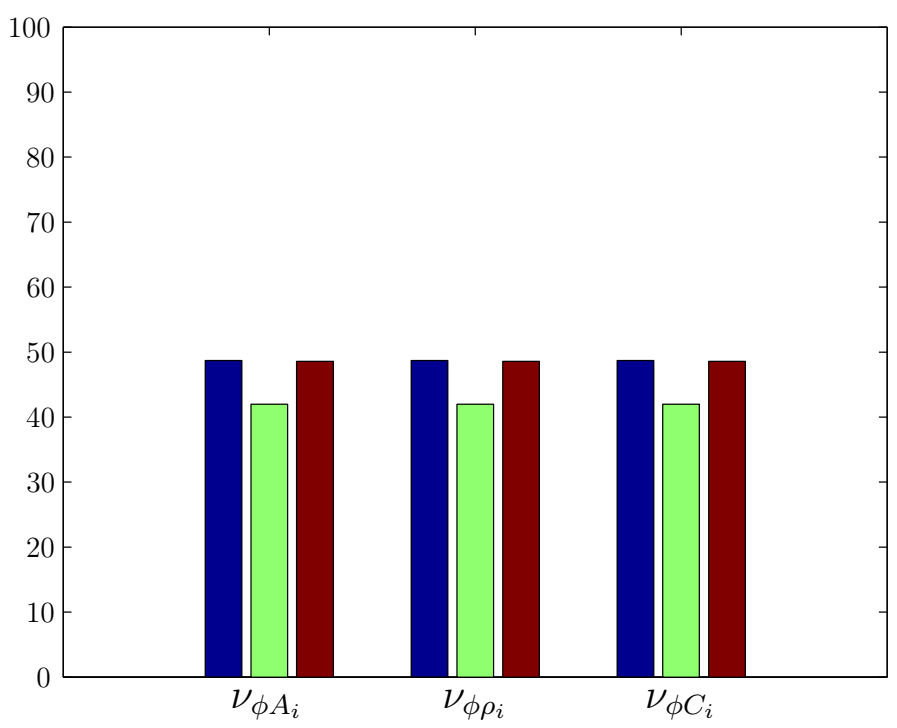

(a)

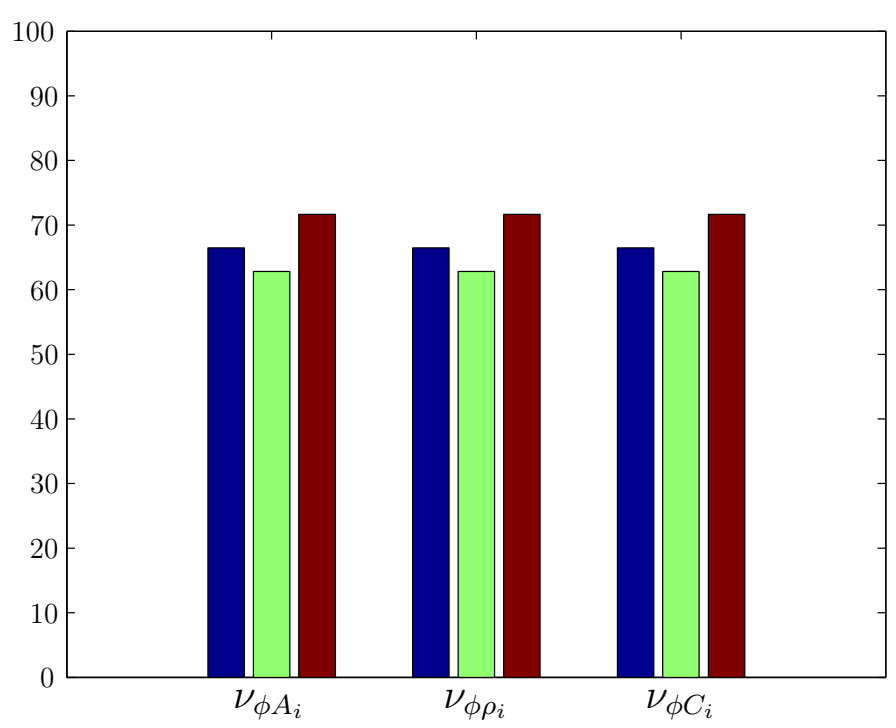

(b)

Figure 5. The percentage of $\mathcal{W}_{s}$, in which: (a) $v_{\phi q_{i}}<3 \mathrm{rad} / \mathrm{m}$; (b) $v_{\phi q_{i}}<6 \mathrm{rad} / \mathrm{m}, q_{i}=\left\{A_{i}, \rho_{i}, C_{i}\right\}, i=1,2,3$

respectively. For the first set of three bars, $q_{i}$ stands for $A_{i}$. For the second set of three bars, $q_{i}$ stands for $\rho_{i}$. For the third set of three bars, $q_{i}$ stands for $C_{i}, i=1,2,3$. It is apparent that the higher the bar, the smaller the sensitivity of the MP orientation to variations in the corresponding geometric parameter or variable. For instance, from Fig. 5(a), $v_{\phi A_{1}}$ is smaller than $3 \mathrm{rad} / \mathrm{m}$ in $49.3 \%$ of $\mathcal{W}_{s}, v_{\phi \rho_{2}}$ is smaller than $3 \mathrm{rad} / \mathrm{m}$ in $42.4 \%$ of $\mathcal{W}_{s}$ and $v_{\phi C_{3}}$ is smaller than $3 \mathrm{rad} / \mathrm{m}$ in $48.9 \%$ of $\mathcal{W}_{s}$.

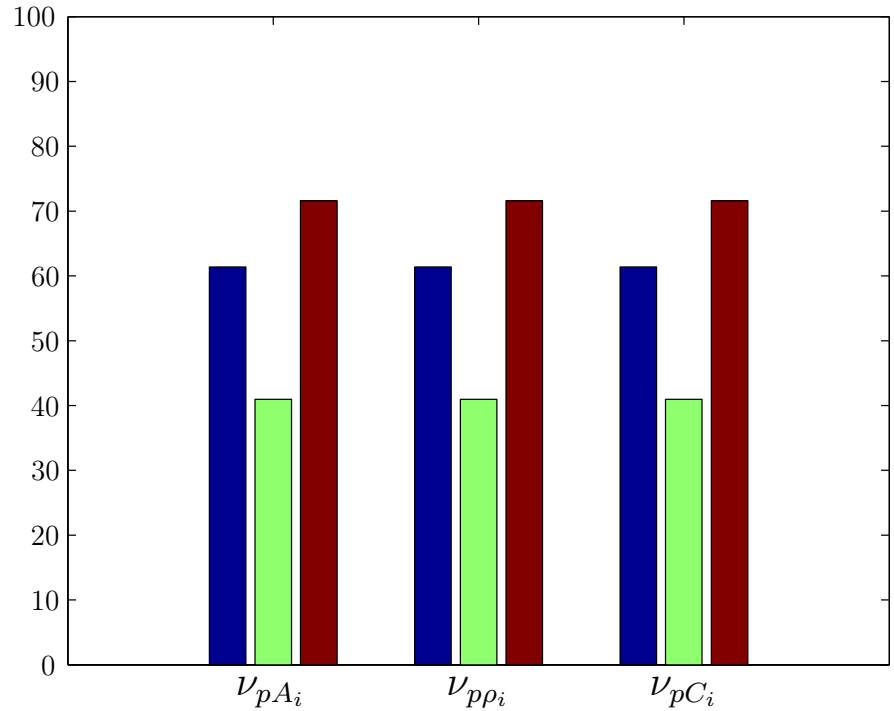

(a)

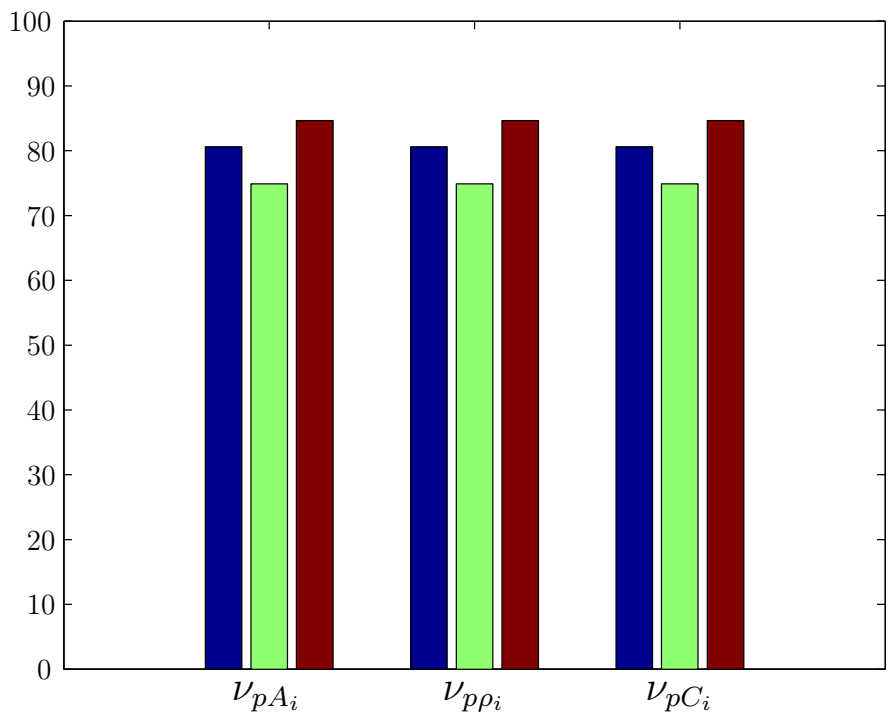

(b)

Figure 6. The percentage of $\mathcal{W}_{s}$, in which: (a) $v_{p q_{i}}<1.5$; (b) $v_{p q_{i}}<3, q_{i}=\left\{A_{i}, \rho_{i}, C_{i}\right\}, i=1,2,3$ 
Figures 6(a)-(b) illustrate the percentage of $\mathcal{W}_{s}$, in which the position-sensitivity indices to variations in the coordinates of $A_{i}, C_{i}$ and in $\rho_{i}$, defined with Eqs. (18d)-(f), are smaller than 1.5 and 3, respectively. The three bars above indices $v_{p q_{i}}$, $i=1,2,3$, are associated with the sensitivity of the MP position to variations in $q_{1}, q_{2}$ and $q_{3}$, respectively. For the first set of three bars, $q_{i}$ stands for $A_{i}$. For the second set of three bars, $q_{i}$ stands for $\rho_{i}$. For the third set of three bars, $q_{i}$ stands for $C_{i}, i=1,2,3$. The higher the bar, the smaller the sensitivity of the MP position to variations in the corresponding geometric parameter or variable. For instance, from Fig. 6(a), $v_{p A_{1}}$ is smaller than 1.5 in $61.2 \%$ of $\mathcal{W}_{s}, v_{p \rho_{2}}$ is smaller than 1.5 in $40.6 \%$ of $\mathcal{W}_{s}$ and $v_{p C_{3}}$ is smaller than 1.5 in $71.1 \%$ of $\mathcal{W}_{s}$.

From Figs. 5(a)-(b), we can notice that $v_{\phi A_{i}}, v_{\phi \rho_{i}}$ and $v_{\phi C_{i}}$ are similar. Likewise, from Fig. 5(a)-(b), $v_{p A_{i}}, v_{p \rho_{i}}$ and $v_{p C_{i}}$ are similar. As a matter of fact, $v_{\phi A_{i}}$ and $v_{\phi C_{i}}\left(v_{p A_{i}}\right.$ and $v_{p C_{i}}$, resp.) is the sensitivity of the MP orientation (position) to the most penalizing variation of the corresponding point. Accordingly, the most penalizing variations of points $A_{i}$ and $C_{i}$ are along leg $A_{i} C_{i}, i=1,2,3$.

For the MP orientation depicted in Fig. 目, we can notice from Figs. 5(a)-(b) that the MP orientation is more sensitive to variations in the geometric parameters of the second leg of the manipulator than to variations in its other geometric parameters as the bars associated with the second leg are smaller than the bars associated with the other legs. Likewise, from Figs. 6(a)-(b), the MP position is more sensitive to variations in the geometric parameters of second leg of the manipulator than to variations in its other geometric parameters as the bars associated with the second leg are smaller than the bars associated with the other legs.

In order to have an idea of the sensitivity of the MP pose of the manipulator to variations in its geometric parameters and prismatic actuated joints, Figs. 7(a)-(b) illustrate the isocontours of $v_{\phi}$ and $v_{p}$, defined with Eqs. (24) and (25), throughout $\mathcal{W}_{s}$. We can notice that the closer $P$ to the geometric center of $\mathcal{W}_{s}$, the smaller the sensitivity of the MP pose to variations in the geometric parameters and prismatic actuated joints.

Figures 8(a)-(b) illustrate the distribution of $v_{\phi}$ and $v_{p}$ throughout $\mathcal{W}_{s}$. For instance, Fig. $8(\mathrm{~b})$ shows that $v_{\phi}$ is lower than $0.4 \mathrm{rad} / \mathrm{m}$ in $24.7 \%$ of $\mathcal{W}_{s}$. Likewise, Fig. 8(a) shows that $v_{p}$ is lower than 0.2 in $32.8 \%$ of $\mathcal{W}_{s}$.

Let us assume that the variations in the geometric parameters and prismatic actuated joints follow a normal distribution and their tolerance range is equal to $50 \mu \mathrm{m}$, namely,

$$
\Delta q_{i}=3 \sigma_{q_{i}}=50 \mu \mathrm{m}, q_{i}=\left\{A_{i}, \rho_{i}, C_{i}\right\}, i=1,2,3
$$



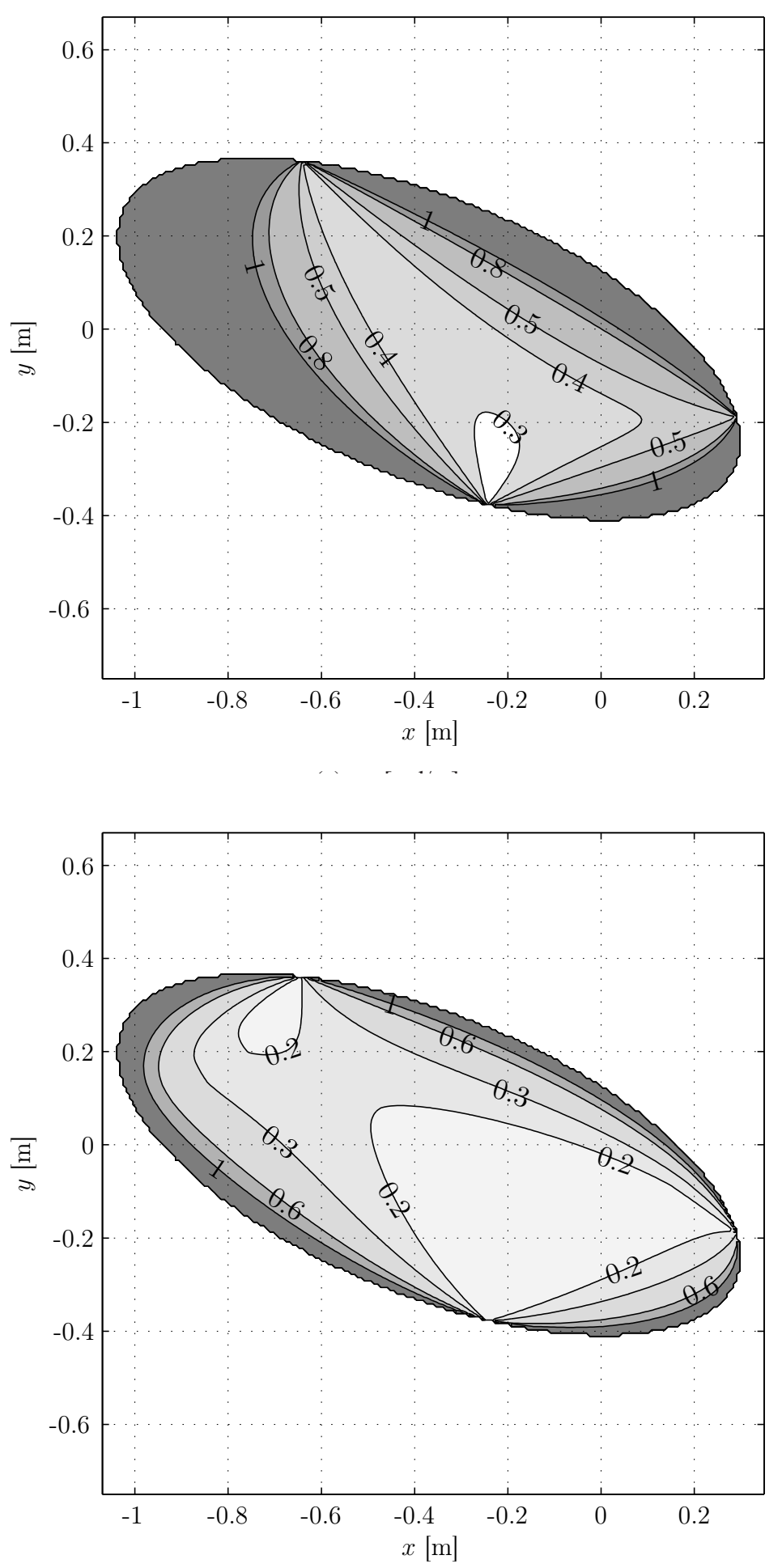

(b) $v_{p}[\mathrm{~m} / \mathrm{m}]$

Figure 7. $v_{\phi}$ and $v_{p}$ isocontours throughout $\mathcal{W}_{s}$ 


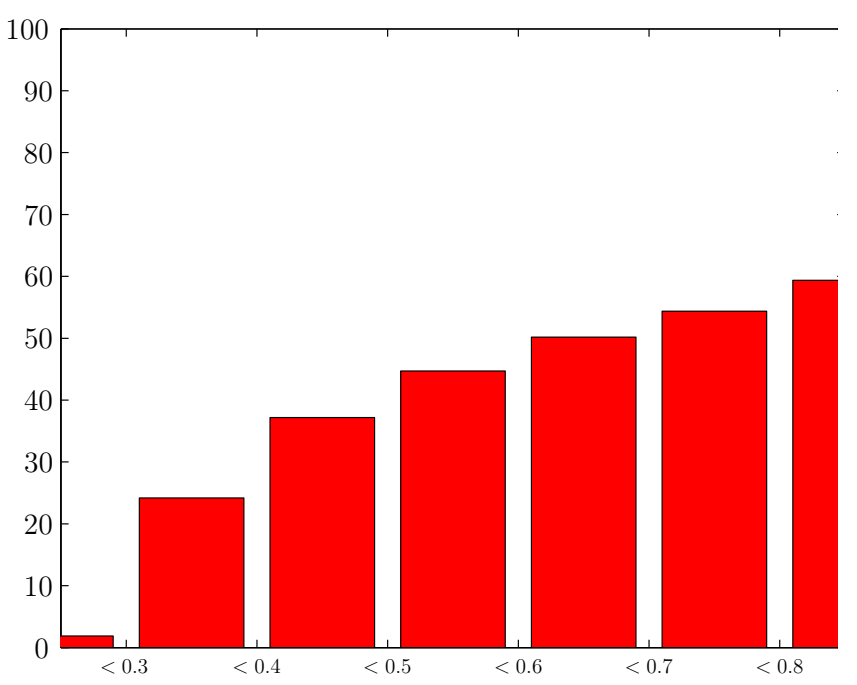

(a) $v_{\phi}$

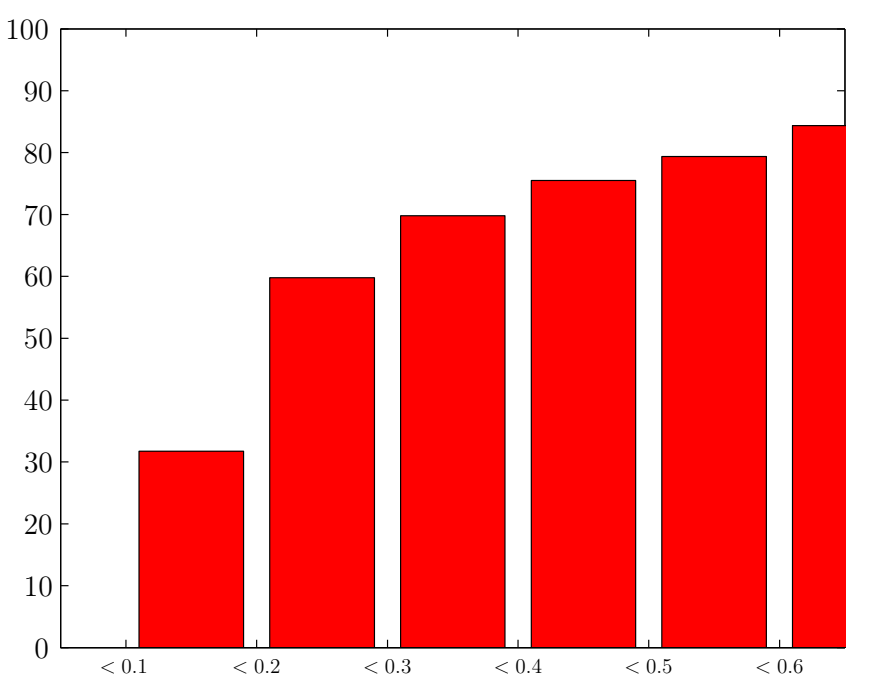

(b) $v_{p}$

Figure 8. Distribution of $v_{\phi}$ and $v_{p}$ throughout $\mathcal{W}_{s}$

$\Delta q_{i}$ and $\sigma_{q_{i}}$ being the tolerance range and the standard deviation of entity $q_{i}, q_{i}=\left\{A_{i}, \rho_{i}, C_{i}\right\}, i=1,2,3$, respectively. Let $|\delta \phi|$

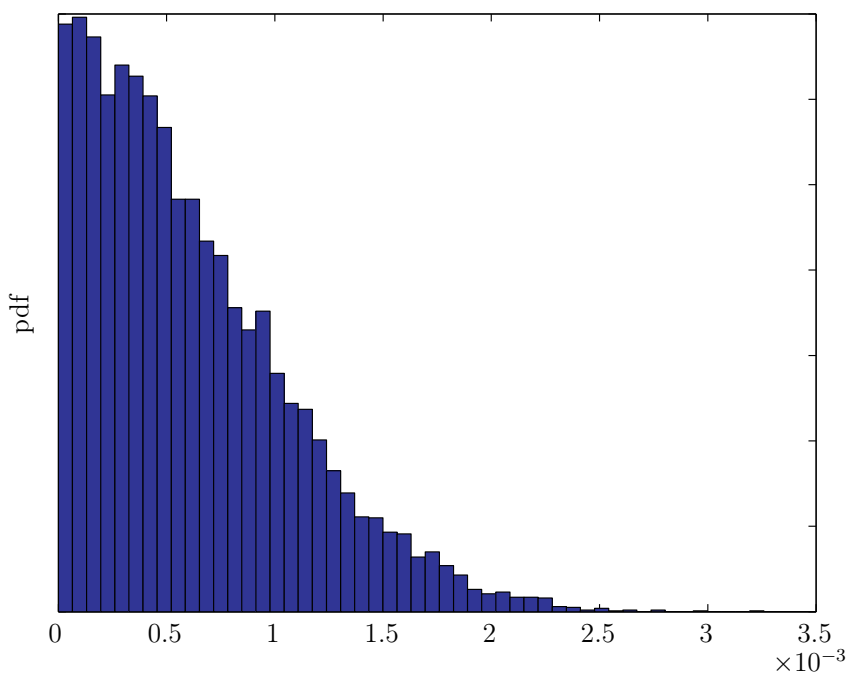

$|\delta \phi|[\mathrm{rad}]$

(a)

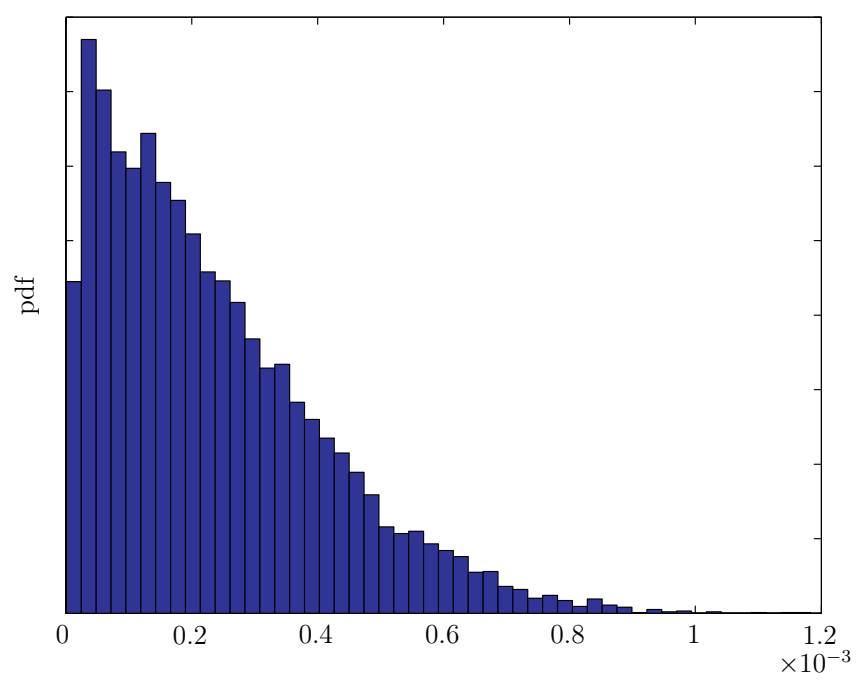

$\|\delta \mathbf{p}\|_{2}[\mathrm{~m}]$

(b)

Figure 9. Distribution of the (a) orientation and (b) positioning errors of the MP for a given pose of the latter: $\phi=\pi / 8$ and $\mathbf{p}=[0.25,0.4]$

and $\|\delta \mathbf{p}\|_{2}$ be the absolute value of the orientation error and the 2-norm of the positioning error of the MP of the manipulator, respectively. Figures (a)-(b) illustrate their distribution evaluated by means of Eq. (19) for the MP pose depicted in Fig. 目 
and the tolerance ranges specified in Eq.(31). Let $|\delta \phi|_{\text {mean }}$ be the average of the absolute orientation error of the MP and $\|\delta \mathbf{p}\|_{2 m e a n}$ the 2-norm of its positioning error. From Figs. $9(\mathrm{a})-(\mathrm{b}),|\delta \phi|_{\text {mean }}$ is equal to $623 \mu \mathrm{rad} / \mathrm{m}$ and $\|\delta \mathbf{p}\|_{2 m e a n}$ is equal to $232 \mu \mathrm{m}$. Figures $10(\mathrm{a})$-(b) show the isocontours of $|\delta \phi|_{\text {mean }}$ and $\|\delta \mathbf{p}\|_{2 \text { mean }}$ throughout $\mathcal{W}_{s}$. Those isocontours are similar to $v_{\phi}$ and $v_{p}$ isocontours illustrated in Figs. 7(a)-(b). It means that $v_{\phi}$ and $v_{p}$ are relevant sensitivity indices of the MP pose to variations in the geometric parameters and in actuated variables.

\subsection{Comparison of Two Non-Degenerate and Two Degenerate 3-RPR PPMs}

In order to highlight the comparison methodology proposed in Section $\emptyset$, the sensitivity of two degenerate and two nondegenerate 3-RPR PPMs is analyzed. Degenerate manipulators have a simpler direct kinematic characteristic polynomial and simpler singularity conditions. Whether they are globally more or less sensitive to geometric errors than their nondegenerate counterparts is a question of interest for the designer. First, the two degeneracy features are recalled. Then, the architectures of the four manipulators under study are illustrated. Finally, those four manipulators are compared based on the size of their regular dexterous workspace and the sensitivity of their MP pose to variations.

5.2.1 Degeneracy Conditions The forward kinematic problem of a parallel manipulator often leads to complex equations and non analytic solutions, even when considering 3-DOF PPMs [22]. For those manipulators, Hunt showed that the forward kinematics admits at most six solutions [23] and some authors proved that their forward kinematics can be reduced to the solution of a sixth-degree characteristic polynomial [24, 25]. The decreasing conditions of the degree of the latter were investigated in [26], [27] and [28]. Here, we focus on the sensitivity analysis of two classes of degenerate manipulators. The first class includes all 3-RPR manipulators with similar base and moving platforms [27]. As far as the degenerate manipulators of the second class are concerned, their moving platform is obtained from their base platform by means of a reflection [28]. For manipulators of the first class, the forward kinematics is reduced to the solution of two quadratics in cascade. For manipulators of the second class, the forward kinematics degenerates is reduced to the solution of a cubic and a quadratic in sequence.

5.2.2 Manipulators Under Study Figures 11(a)-(d) illustrate the four manipulators under study, before geometric parameters normalization, named $M_{1}, M_{2}, M_{3}$ and $M_{4}$, respectively. $M_{1}$ and $M_{2}$ are non-degenerate whereas $M_{3}$ and $M_{4}$ are degenerate. In Fig. 11(a), it is apparent that the base and moving platforms of $M_{1}$ are equilateral. From Fig. 11(b), the base and moving platforms of $M_{2}$ are identical but in a different geometric configuration for an orientation $\phi=0 . M_{3}$ and 

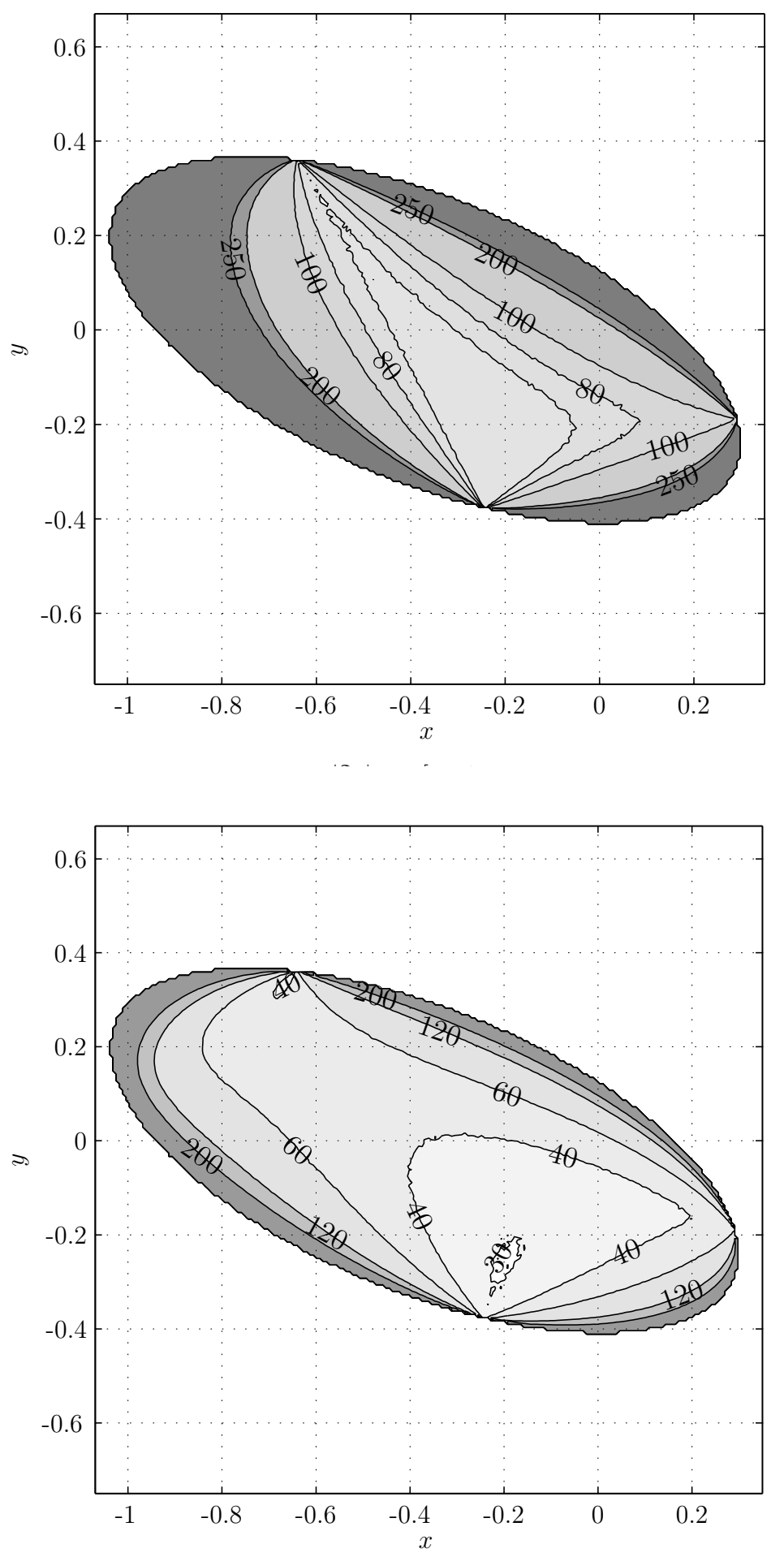

(b) $\|\delta \mathbf{p}\|_{2 m e a n}[\mu \mathrm{m}]$

Figure 10. (a) $|\delta \phi|_{\text {mean }}$ and (b) $\|\delta \mathbf{p}\|_{2 \text { mean }}$ isocontours throughout $\mathcal{W}_{s}$ 
$M_{4}$ illustrate two degeneracy cases. It is noteworthy that the base and moving platforms of $M_{2}, M_{3}$ and $M_{4}$ have the same circumscribed circle, its radius being equal to 1 . As far as $M_{1}$ is concerned, the circumscribed circle of its moving platform is two times smaller than the one of the base platform. With the geometric parameters normalization introduce in Section 4.1 the sum of their radius being is equal to 2 .

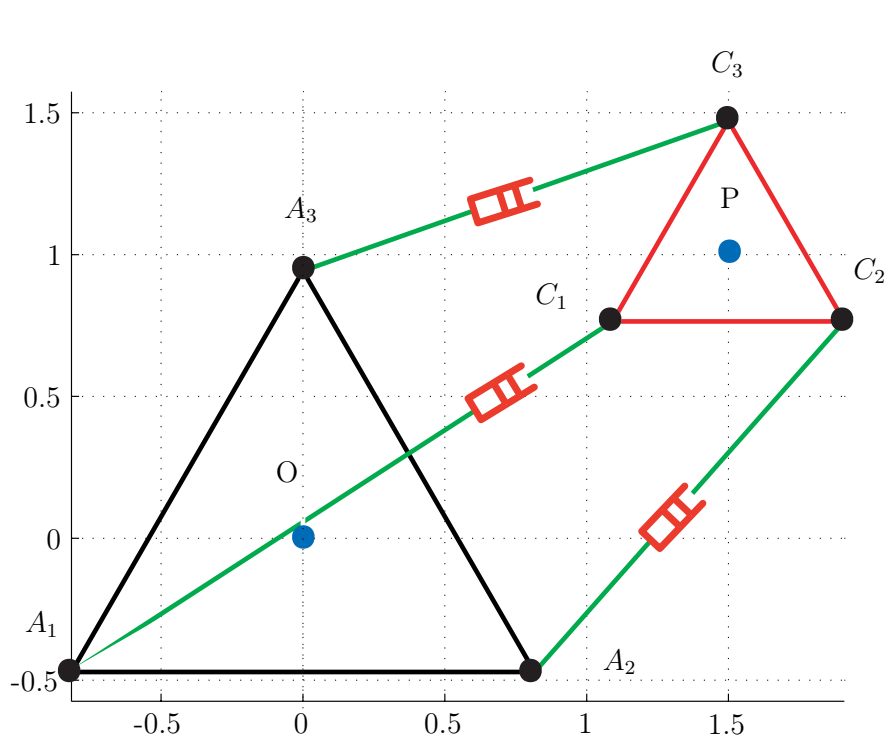

(a) $M_{1}$

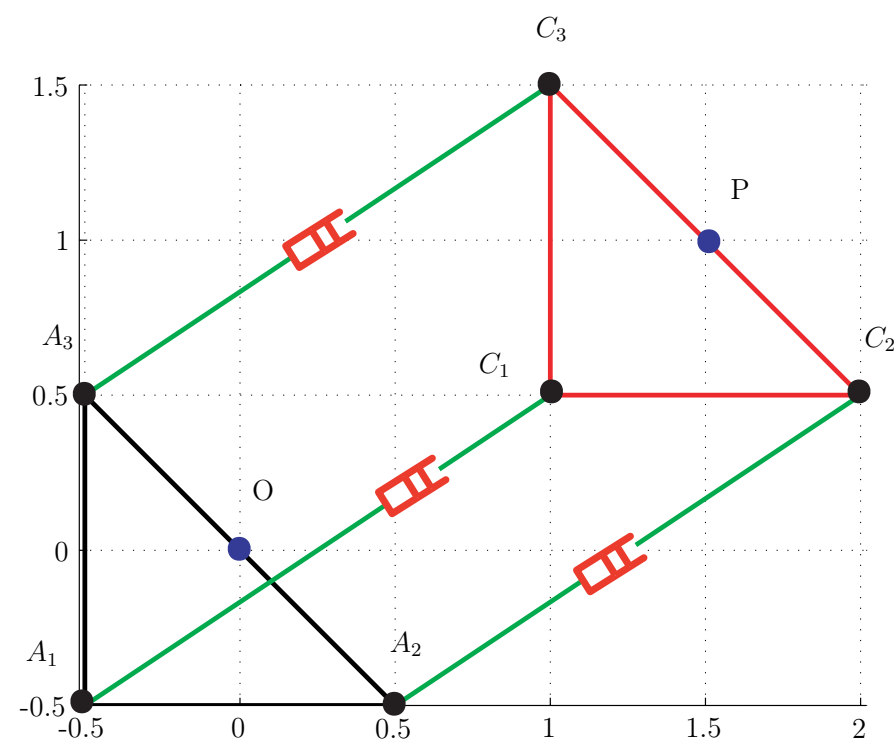

(c) $M_{3}$

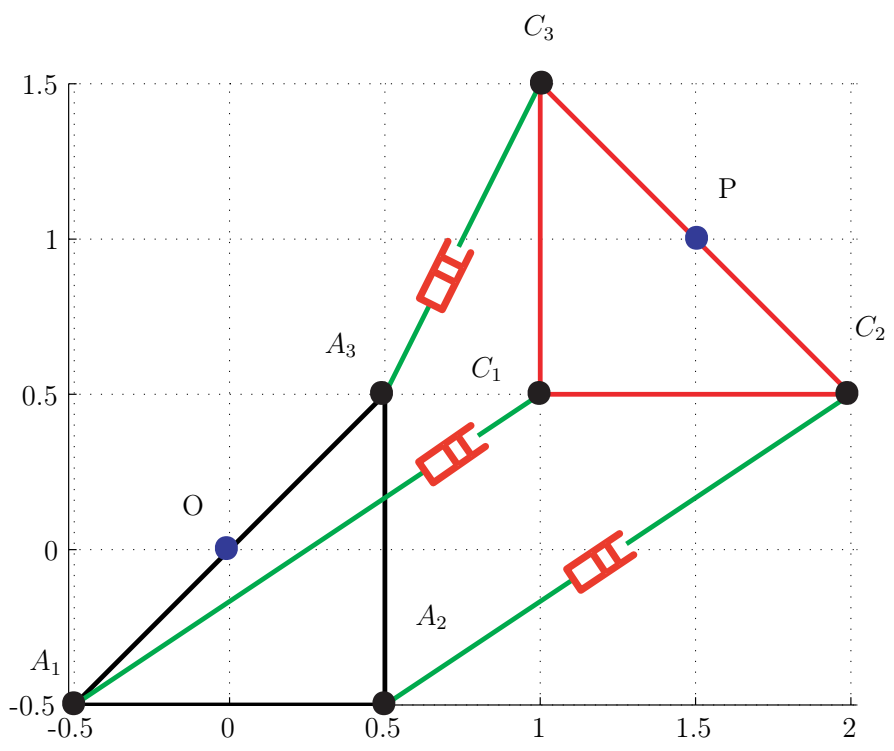

(b) $M_{2}$

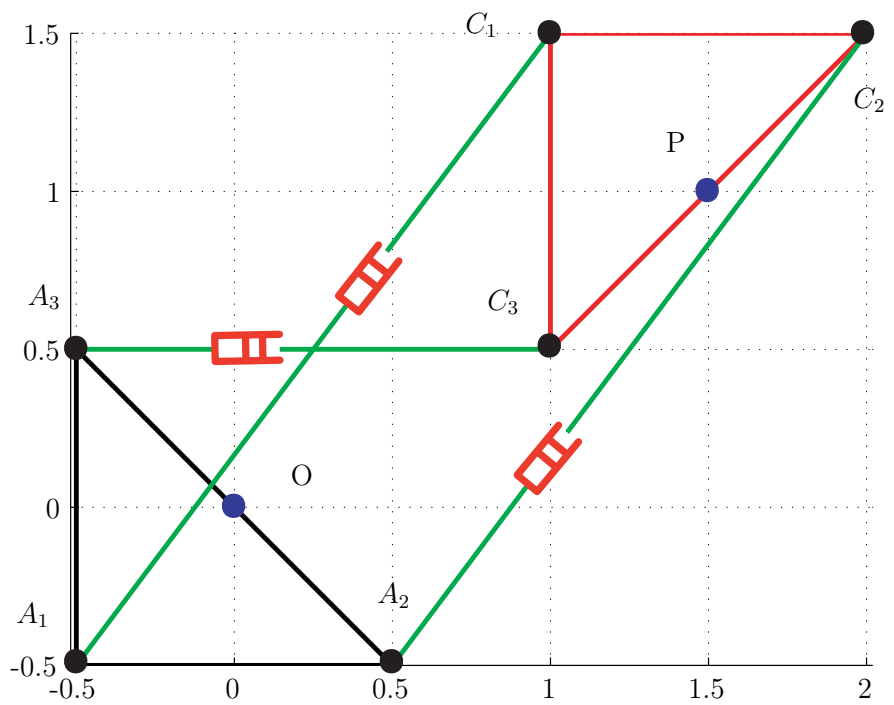

(d) $M_{4}$

Figure 11. The four 3-R $\underline{P R}$ manipulators under study with $\phi=0$ and $\mathbf{p}=[1,1.5]^{T}$ : (a)-(b) non-degenerate manipulators, (c)-(d) degenerate manipulators 
5.2.3 Regular Dexterous Workspace In order to compare the sensitivity of the foregoing manipulators, we first define their RDW as defined in subsection 4.2. Then, the sensitivity of $M_{1}, M_{2}, M_{3}$ and $M_{4}$ can be evaluated throughout their RDW and compared. Figures 12(a)-(d) illustrate the workspace window equal to $x=[-2.5 ; 2.5], y=[-2.5 ; 2.5]$ and $\phi=[-\pi ; \pi]$, the singularity surfaces and the maximal RDW of $M_{1}, M_{2}, M_{3}$ and $M_{4}$. Their radii are given in Table 11 and compared in Fig. 13. We can notice that $M_{4}$ has the largest RDW, whereas $M_{2}$ has the smallest one.

\begin{tabular}{cccc}
\hline \hline$R_{1}$ & $R_{2}$ & $R_{3}$ & $R_{4}$ \\
1.18 & 0.64 & 0.92 & 1.43 \\
Table 1. RDW radius of $M_{1}, M_{2}, M_{3}$ and $M_{4}$
\end{tabular}

5.2.4 $v_{\phi}$ and $v_{p}$ Isocontours In this section, the sensitivity of $M_{1}, M_{2}, M_{3}$ and $M_{4}$ is evaluated within their RDW for a matter of comparison based on aggregate sensitivity indices $v_{\phi}$ and $v_{p}$ defined with Eqs.(24) and (25), respectively.

Figures 14(a)-(d) (Figures 15(a)-(d), resp.) illustrate the isocontours of the maximum value of $v_{\phi}\left(v_{p}\right.$, resp.) for a given orientation $\phi$ of the MP throughout the RDW of $M_{1}, M_{2}, M_{3}$ and $M_{4}$, respectively. It is apparent that $M_{4}$ has the least sensitive orientation of its MP whereas $M_{1}$ has the least sensitive position of its MP to variations in geometric parameters. On the contrary, $M_{1}$ has the most sensitive position of its MP and $M_{4}$ has the most sensitive orientation of its MP to variations in geometric parameters.

Figures 16(a)-(b) show the distributions of $v_{\phi}$ and $v_{p}$ throughout the RDW of $M_{1}, M_{2}, M_{3}$ and $M_{4}$. From Fig. 16(a), $v_{\phi}$ is smaller than 0.3 in $78.4 \%(93.7 \%, 90.3 \%$ and $98.3 \%$, resp. $)$ of $M_{1}\left(M_{2}, M_{3}\right.$ and $M_{4}$, resp.) RDW. From Fig. 16(b), $v_{p}$ is smaller than 0.2 in $79.9 \%$ (48.8\%, $78.4 \%$ and $18.7 \%$, resp.) of $M_{1}\left(M_{2}, M_{3}\right.$ and $M_{4}$, resp.) RDW.

Finally, Table 1 gives an overall classification of $M_{1}, M_{2}, M_{3}$ and $M_{4}$ with regard to their RDW size and the sensitivity of their MP orientation and position to variations in their geometric parameters. We can notice that the degenerate manipulator $M_{4}$ is globally the most interesting, i.e., it has the most robust design. The sensitivity analysis of these four manipulators has been carried out with other RDWs, i.e., with different upper bounds of $\Delta \phi$ and $\kappa_{F}^{-1}\left(\mathbf{J}_{n}\right)$ in the optimization problem formulated in Section 4.2. The results are reported in [29] and it turns out that the overall classification shown in Table 2 is unchanged. 


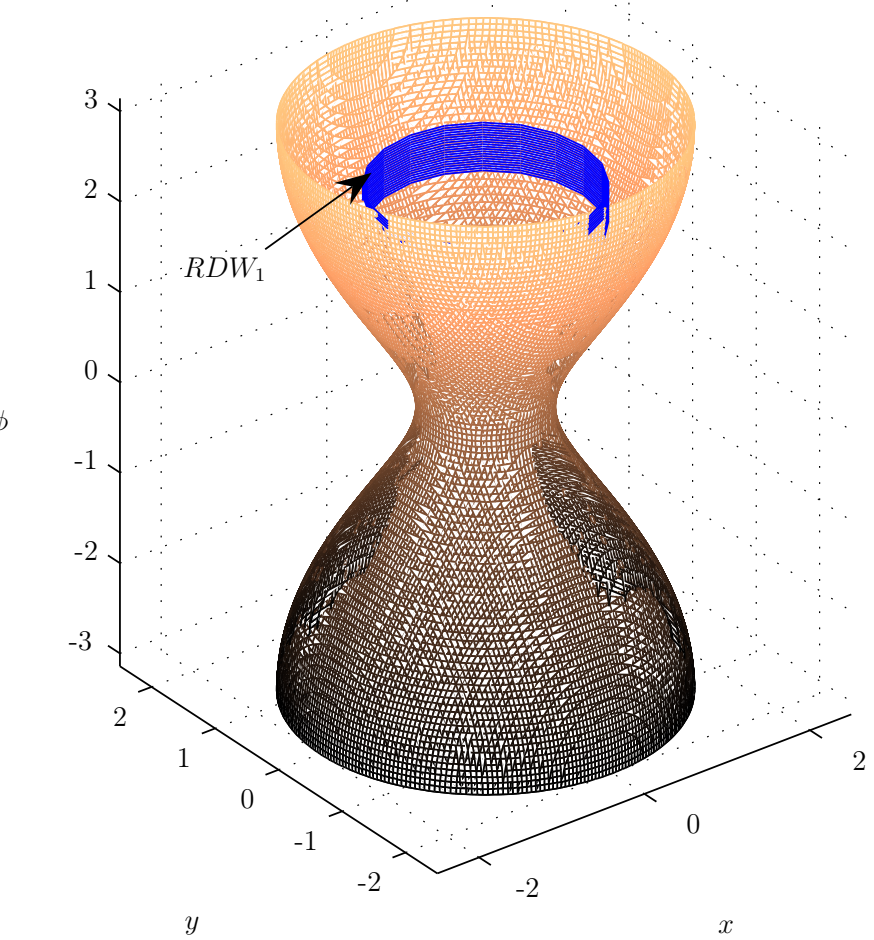

(a) $M_{1}$

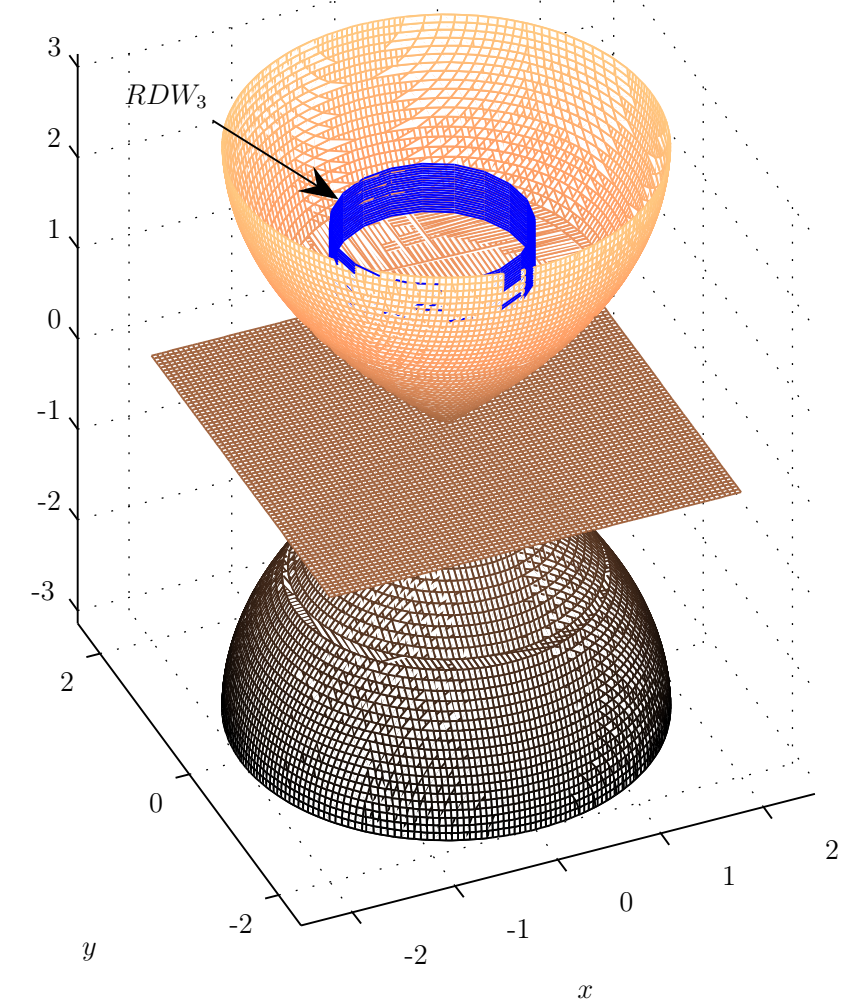

(c) $M_{3}$

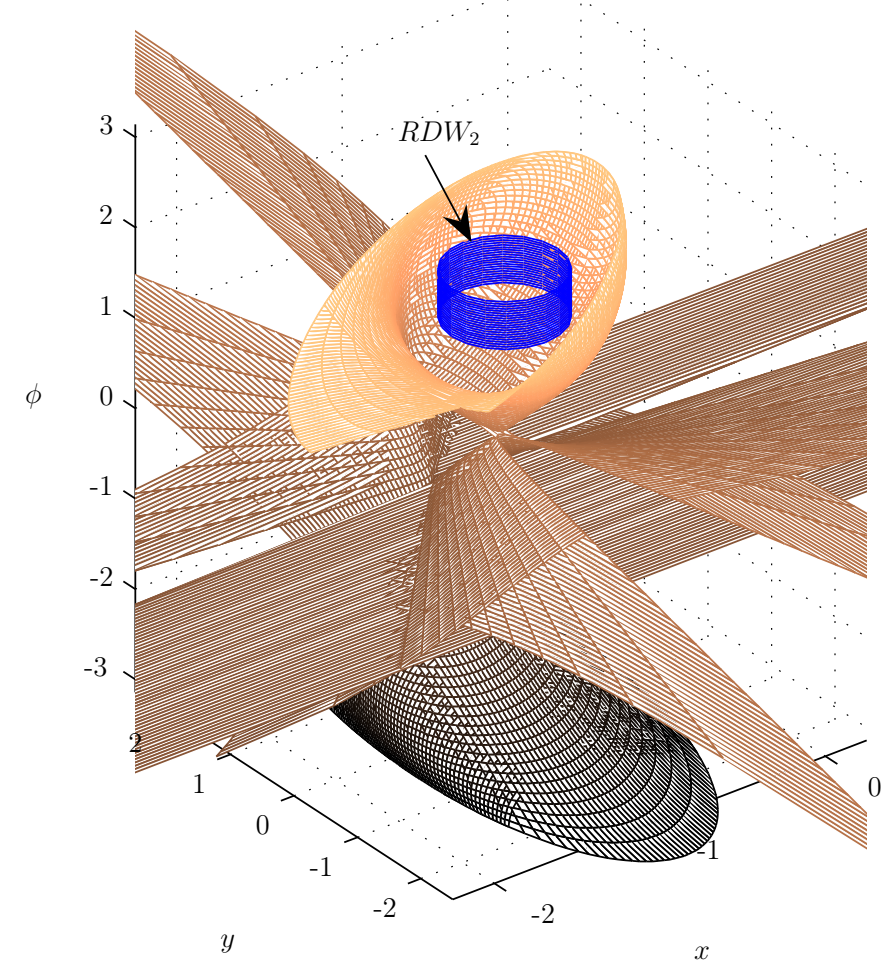

(b) $M_{2}$

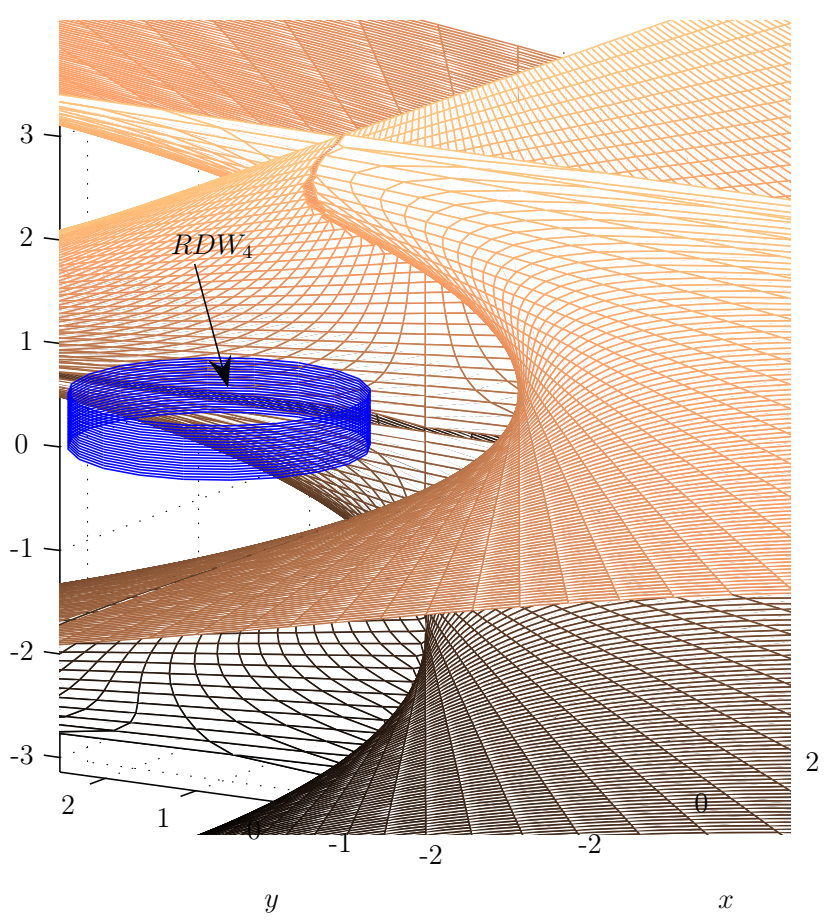

(d) $M_{4}$

Figure 12. Maximal Regular Dextrous Workspace of: (a) $M_{1}$; (b) $M_{2}$; (c) $M_{3}$ and (d) $M_{4}$ 


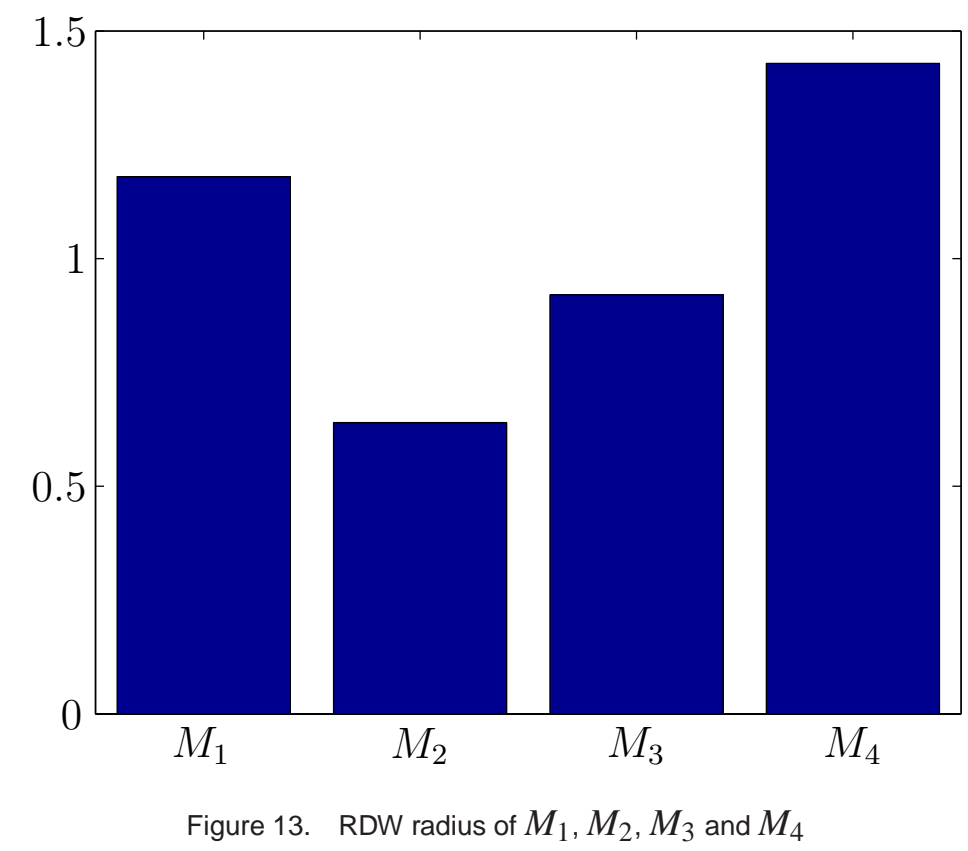

\begin{tabular}{c|cccc}
\hline \hline & $M_{1}$ & $M_{2}$ & $M_{3}$ & $M_{4}$ \\
\hline$R D W$ & 2 & 4 & 3 & 1 \\
$v_{\phi}$ & 4 & 3 & 2 & 1 \\
$v_{p}$ & 1 & 3 & 2 & 4 \\
\hline Ranking & 2 & 4 & 2 & 1 \\
\hline \hline
\end{tabular}

Table 2. Classification of $M_{1}, M_{2}, M_{3}$ and $M_{4}$ w.r.t $v_{\phi}, v_{p}$ and their RDW size

\section{CONCLUSIONS}

This paper dealt with the sensitivity analysis of 3-Rㅛ planar parallel manipulators (PPMs). First, the sensitivity coefficients of the pose of the manipulator moving platform to variations in the geometric parameters and in the actuated variables were expressed algebraically. Moreover, two aggregate sensitivity indices were determined, one related to the orientation of the moving platform of the manipulator and another one related to its position. Then, a methodology was proposed to compare 3-Rㅛ PPMs with regard to their dexterity, workspace size and sensitivity. The sensitivity of a 3RPR PPM was analyzed in detail and four 3-RPR PPMs were compared as illustrative examples. The sensitivity indices $v_{\phi}$ and $v_{p}$ introduced in the paper should help the designer of 3-R PR PPMs at their conceptual design stage. The actuated joint limits were not considered in this study, but have to be used for the determination of the manipulator size. As a matter of fact, they can be calculated knowing the location and the size of the maximal RDW. In order to deal with this 


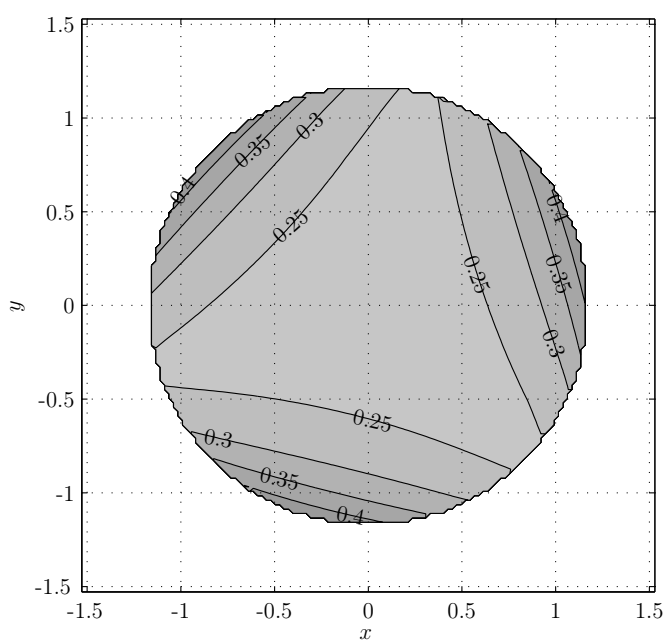

(a) $M_{1}: v_{\mathrm{h}}$

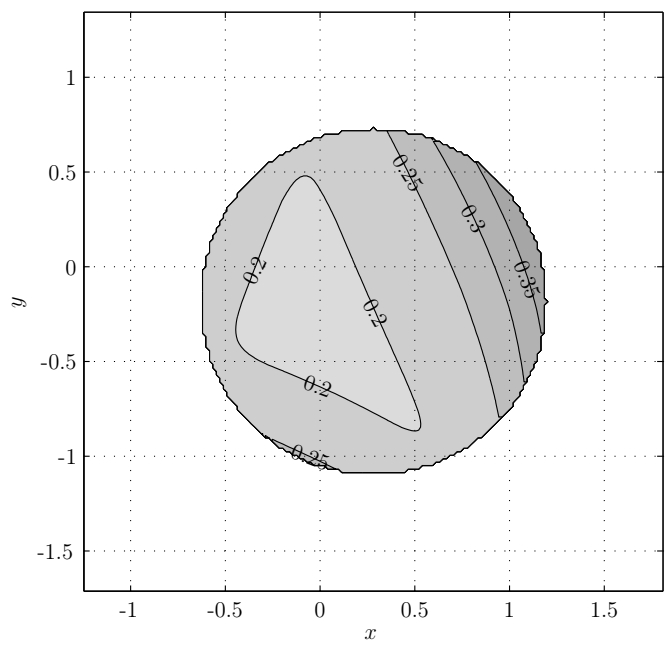

(c) $M_{3}: v_{\phi}$

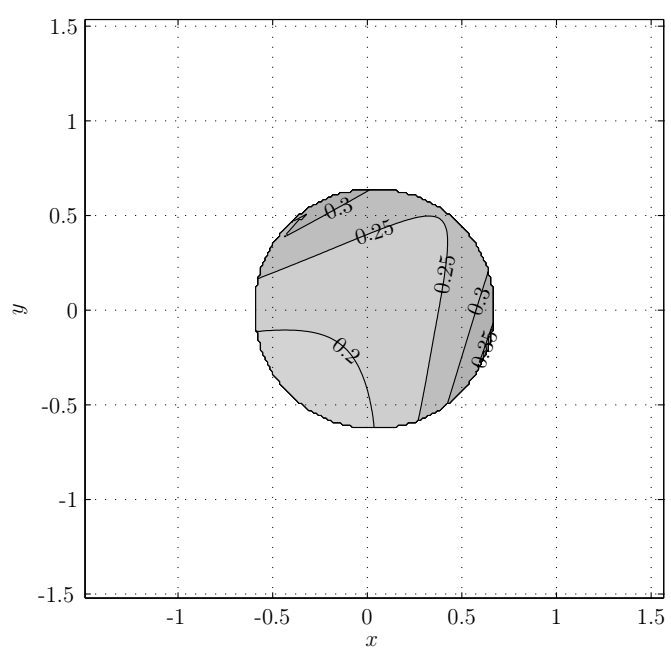

(b) $M_{7}: v_{\mathrm{h}}$

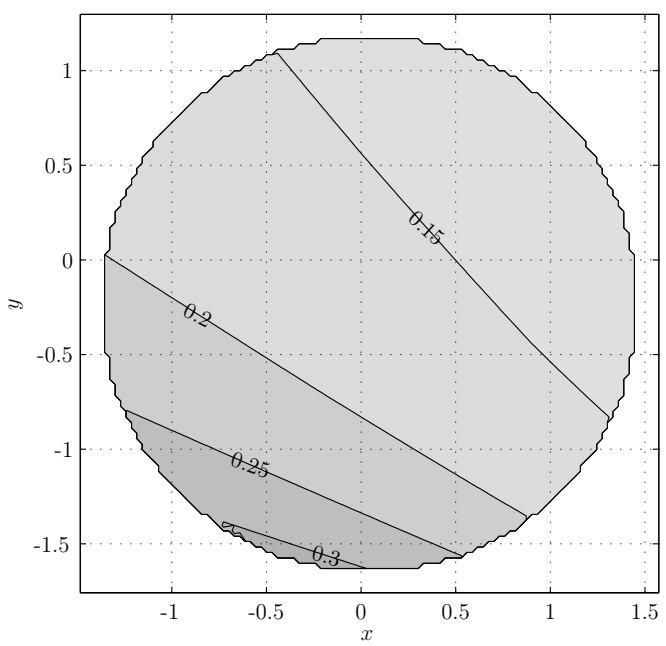

(d) $M_{4}: v_{\phi}$

Figure 14. $v_{\phi}$ isocontours of (a) $M_{1}$, (b) $M_{2}$, (c) $M_{3}$ and (d) $M_{4}$

problem, the RDW can be plotted in the joint space and its smallest enveloping parallelepiped be determined. Later on, the methodology proposed in this paper will be used to compare the sensitivity of PPMs of different architectures and/or dimensions to variations in their geometric parameters.

\section{REFERENCES}

[1] Wang, J. and Masory, O. (1993). "On the Accuracy of a Stewart Platform - Part I, The Effect of Manufacturing Tolerances," In: Proceedings of the IEEE International Conference on Robotics Automation, ICRA'93, Atlanta, USA, pp. 114-120. 


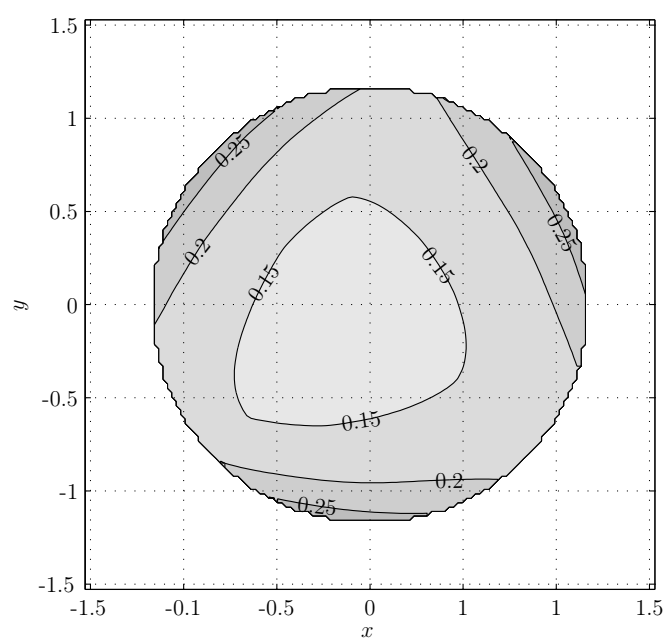

(a) $M_{1}: v_{n}$

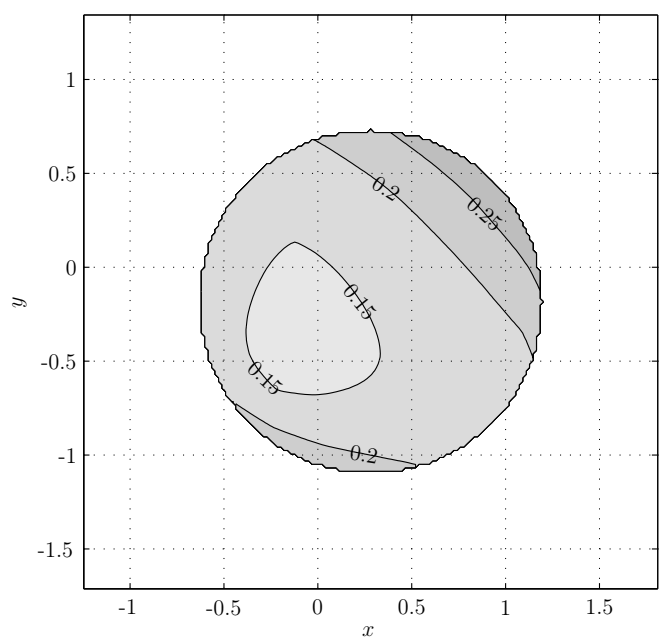

(c) $M_{3}: v_{p}$

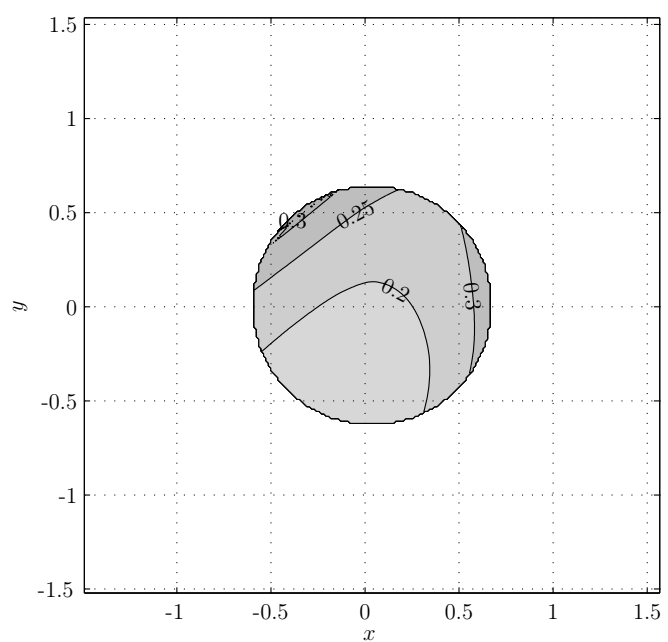

(b) $M_{0}: v_{n}$

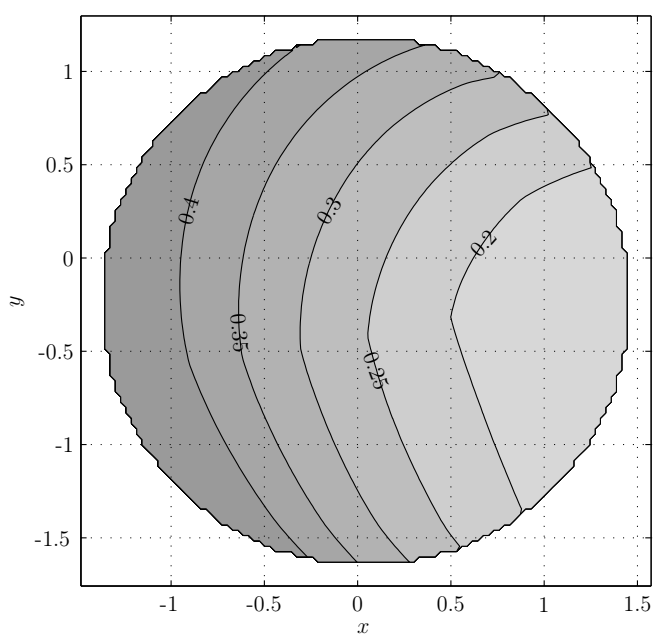

(d) $M_{4}: v_{p}$

Figure 15. $\mathrm{v}_{p}$ isocontours of (a) $M_{1}$, (b) $M_{2}$, (c) $M_{3}$ and (d) $M_{4}$

[2] Kim, H.S. and Choi, Y.J. (2000). “The Kinematic Error Bound Analysis of the Stewart Platform,” Journal of Robotic Systems, 17, pp. 63-73.

[3] Kim, H.S. and Tsai, L-W. (2003). "Design Optimization of a Cartesian Parallel Manipulator," ASME Journal of Mechanical Design, 125, pp. 43-51.

[4] Caro, S., Bennis, F. and Wenger, P. (2005). “Tolerance Synthesis of Mechanisms: A Robust Design Approach,” ASME Journal of Mechanical Design, 127, January, pp. 86-94.

[5] Caro, S., Wenger, P., Bennis, F. and Chablat, D. (2006). "Sensitivity Analysis of the Orthoglide, A 3-DOF Translational Parallel Kinematics Machine,” ASME Journal of Mechanical Design, 128, March, pp. 392-402. 


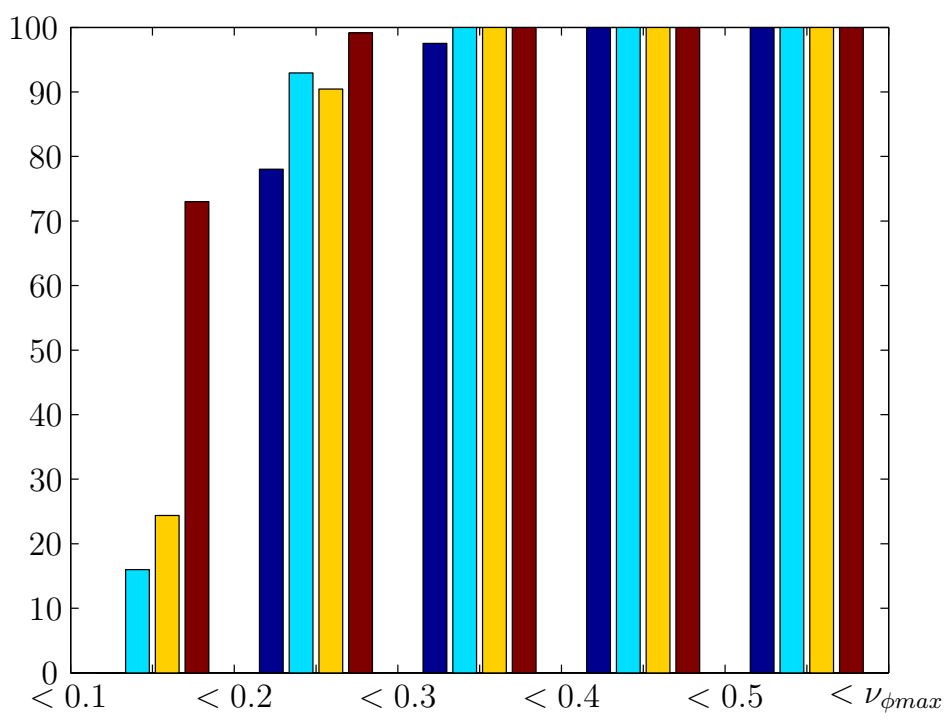

(a) $v_{\phi}$

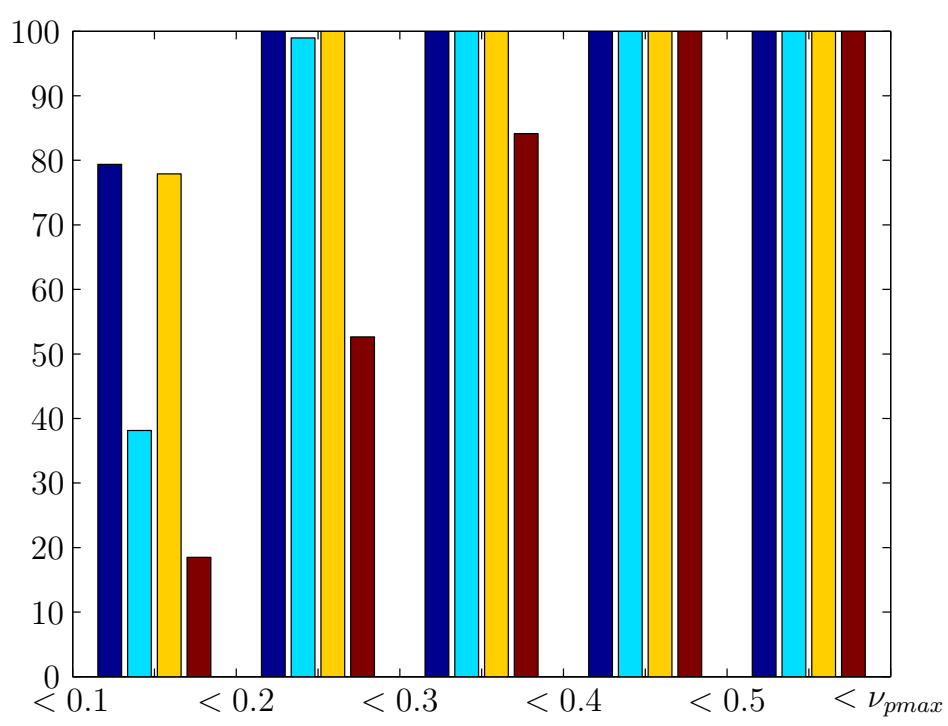

(b) $v_{p}$

Figure 16. Distribution of $\mathrm{v}_{\phi}$ and $\mathrm{v}_{p}$ throughout the RDW of $M_{1}, M_{2}, M_{3}$ and $M_{4}$

[6] Yu, A., Bonev, I.A. and Zsombor-Murray, P.J. (2007). "Geometric Method for the Accuracy Analysis of a Class of 3-DOF Planar Parallel Robots,” Mechanism and Machine Theory, 43(3), pp. 364-375.

[7] Meng, J., Zhang, D. and Li, Z. (2009). “Accuracy Analysis of Parallel Manipulators With Joint Clearance," ASME Journal of Mechanical Design, 131, January, pp. 011013-1-011013-9.

[8] Han, C., Kim, J., Kim, J. and Chongwoo Park, F. (2002). "Kinematic Sensitivity Analysis of the 3-UPU Parallel Mechanism," Mechanism and Machine Theory, 37(8), pp. 787-798. 
[9] Bonev, I.A., Zlatanov, D. and Gosselin, C.M. (2003). "Singularity Analysis of 3-DOF Planar Parallel Mechanisms via Screw Theory,” ASME Journal of Mechanical Design, 125, pp. 573-581.

[10] Merlet, J.P. (2006). Parallel robots, 2nd ed., Springer.

[11] Gosselin, C.M. and Angeles, J. (1990). "Singularity Analysis of Closed-Loop Kinematic Chains,” IEEE Transactions on Robotics and Automatics, 6(3), pp. 281-290.

[12] Liu, X.-J., Wang, J. and Pritschow, G. (2006). "Kinematics, Singularity and Workspace of Planar 5R Symmetrical Parallel Mechanisms," Mechanism and Machine Theory, 41(2), pp. 145-169.

[13] Liu, X.-J., Wang, J. and Pritschow, G. (2006). "Performance Atlases and Optimum Design of Planar 5R Symmetrical Parallel Mechanisms," Mechanism and Machine Theory, 41(2), pp. 119-144.

[14] Liu, X.-J., Wang, J. and Pritschow, G. (2006). "On the Optimal Design of the PRRRP 2-DOF Parallel Mechanism," Mechanism and Machine Theory, 41(9), pp. 1111-1130.

[15] Merlet, J.P. (2006). "Jacobian, Manipulability, Condition Number, and Accuracy of Parallel Robots," ASME Journal of Mechanical Design, 128, pp. 199-206.

[16] Caro, S., Chablat, D., Wenger, P. and Angeles, J. (2003). "Isoconditioning Loci of Planar Three-Dof Parallel Manipulators,” In: G. Gogu, D. Coutellier, P. Chedmail and P. Ray (Editors), Recent Advances in Integrated Design and Manufacturing in Mechanical Engineering, Kluwer Academic Publisher, 2003, pp. 129-138.

[17] Alba-Gomez, O., Wenger, P. and Pamanes, A. (2005). “Consistent Kinetostatic Indices for Planar 3-DOF Parallel Manipulators, Application to the Optimal Kinematic Inversion," ASME Design Engineering Technical Conferences, September, Long Beach, U.S.A.

[18] Chablat, D., Wenger P., Majou F. and Merlet, J.P. (2006). “An Interval Analysis Based Study for the Design and the Comparison of 3-DOF Parallel Kinematic Machines," International Journal of Robotics Research, 23(6), pp. 615-624.

[19] Khan, W.A. and Angeles, J. (2006). “The Kinetostatic Optimization of Robotic Manipulators: The Inverse and the Direct Problems,” ASME Journal of Mechanical Design, 129, pp. 168-178.

[20] Al-Sultan, K.S. and Al-Fawzan, M.A. (1997). "A Tabu Search Hooke and Jeeves Algorithm for Unconstrained Optimization," European Journal of Operational Research, 103, pp. 198-208.

[21] Li, H., Gosselin, C. and Richard, M.J. (2006). "Determination of Maximal Singularity-free Zones in the Workspace of Planar Three-degree-of-freedom Parallel Mechanisms,” Mechanism and Machine Theory, 41(10), pp. 1157-1167.

[22] Hunt, K.H. (1978). Kinematic Geometry of Mechanisms, Oxford University Press, Cambridge. 
[23] Hunt, K.H. (1983). "Structural Kinematics of In-Parallel Actuated Robot Arms," J. of Mechanisms, Transmissions and Automation in Design, 105(4), pp. 705-712.

[24] Gosselin, C., Sefrioui, J. and Richard M. J. (1992). "Solutions Polynomiales au Problème de la Cinématique des Manipulateurs Parallèles Plans Trois Degrés de Liberté,” Mechanism and Machine Theory, 27, pp. 107-119.

[25] Pennock, G.R. and Kassner, D.J. (1990). "Kinematic Analysis of a Planar Eight-Bar Linkage: Application to a Platform-Type Robot," ASME Proc. of the 21th Biennial Mechanisms Conf., Chicago, September, pp. 37-43.

[26] Gosselin, C.M. and Merlet, J-P. (1994). "On the Direct Kinematics of Planar Parallel Manipulators: Special Architectures and Number of Solutions," Mechanism and Machine Theory, 29(8), pp. 1083-1097.

[27] Kong, X. and Gosselin, C.M. (2001). "Forward Displacement Analysis of Third-Class Analytic 3-RPR Planar Parallel Manipulators," Mechanism and Machine Theory, 36(9), pp. 1009-1018.

[28] Wenger, P., Chablat D. and Zein, M. (2007). "Degeneracy Study of the Forward Kinematics of Planar 3-RPR Parallel Manipulators," ASME Journal of Mechanical Design, 129, pp. 1265-1268.

[29] Binaud, N. (2009). “Sensitivity Comparison of Planar Parallel Manipulators,” IRCCyN Internal Report. 